[This paper has been accepted for publication. Published version may differ.]

\title{
Evaluation institutionnelle des actions de la Commission en matière d'aides d'Etat contre la concurrence fiscale passive
}

\begin{abstract}
Résumé
Au sein de l'Union Européenne, les Etats membres ont généralement conservé les compétences dans le domaine de la taxation directe et les utilisent pour attirer les investissements, créant ainsi une concurrence sur le marché du droit fiscal. Par le passé, cette concurrence fiscale s'est manifestée sous la forme d'actions positives des Etats (ex. introduction de régimes fiscaux favorables pour certaines catégories d'entreprises ou d'activités) auxquelles les contribuables réagissaient en planifiant leurs activités. On observe que cette concurrence fiscale a évolué afin d'englober une forme différente, qui se caractérise par l'attitude (trop) passive d'un Etat membre vis-à-vis de la planification fiscale (agressive) des entreprises.

Cette contribution étudie la manière dont ce dernier phénomène de concurrence fiscale passive est appréhendé par le droit européen et en particulier par la réglementation sur les aides d'Etat que la Commission a mobilisée dès 2013 à son encontre. Cette mobilisation a suscité un nombre de critiques, selon lesquelles les actions de la Commission impliquent une interprétation erronée de l'article 107, paragraphe 1 du TFUE sur le fond et en particulier de l'exigence de sélectivité qui bouleversent la structure verticale et horizontale de l'ordre juridique de l'UE. Le but du présent article est d'analyser exclusivement le bien-fondé de cette vague de critiques et, partant, d'évaluer les actions de la Commission d'un point de vue institutionnel.
\end{abstract}

Mots clés : aides d'Etat, fiscalité, droit européen, concurrence fiscale, harmonisation.

\section{Introduction}

Dans l'état actuel du droit de l'Union européenne, les Etats membres ont généralement conservé leurs compétences dans le domaine de la fiscalité directe. Cela conduit de manière inéluctable à une concurrence sur «le marché des droits fiscaux nationaux » pour attirer et garder les investissements. Par le passé, cette concurrence fiscale prenait la forme d'actions positives posées par des Etats Membres qui introduisaient des régimes fiscaux favorables à certaines catégories d'entreprises ou d'activités et auxquelles ces contribuables réagissaient en planifiant leurs activités. Au cours de ces dernières années, on observe que cette concurrence fiscale a également emprunté une autre forme qui se concentre sur la planification fiscale (agressive) des entreprises, vis-à-vis de laquelle un Etat Membre adopte une attitude (trop) passive et ne réagit donc pas (de manière appropriée) (cf. Partie I).

Bien que le domaine de la fiscalité directe relève de la compétence des Etats Membres, il est de jurisprudence constante de la Cour de justice de l'Union européenne (CJUE) que cette compétence doit être exercée conformément au droit de l'Union. Ce dernier inclut évidemment la réglementation sur les aides d'Etat (articles 107-109 TFUE). Si la Commission a traditionnellement utilisé ses prérogatives en matière de concurrence fiscale active entre les Etats membres, depuis 2013, elle a étendu sa pratique en matière d'aides d'Etat aux formes émergentes de concurrence fiscale passive. Ces actions de la Commission ont suscité des critiques, principalement dans la doctrine, en ce sens qu'elle bouleverserait la structure verticale et horizontale de l'ordre juridique de l'UE, ce qui justifie la nécessité d'évaluer le bien-fondé de telles arguments (cf. Partie II). 


\section{Table des matières}

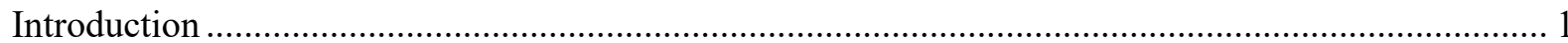

Partie I. L'émergence de la concurrence fiscale passive ....................................................................... 3

Titre 1. Souveraineté des Etats membres en matière de fiscalité directe.......................................... 3

Chapitre 1. Souveraineté fiscale et impôts directs........................................................................ 3

Chapitre 2. La compétence en matière de fiscalité directe n'a pas été transférée à l'UE................ 4

Titre 2. Concurrence fiscale entre les Etats membres ....................................................................... 5

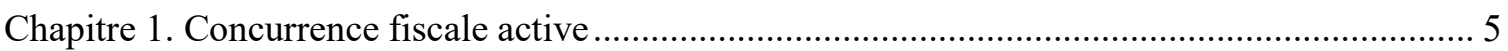

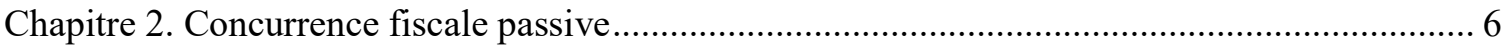

Partie II. L'utilisation du droit des aides d'Etat pour lutter contre la concurrence fiscale passive et son

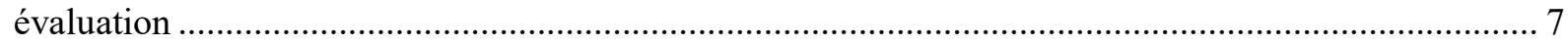

Titre 1. Utilisation du droit des aides d'Etat pour lutter contre la concurrence fiscale passive .......... 9

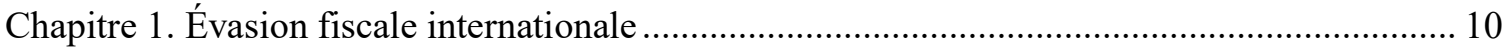

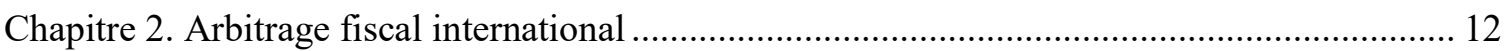

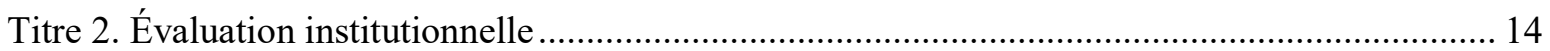

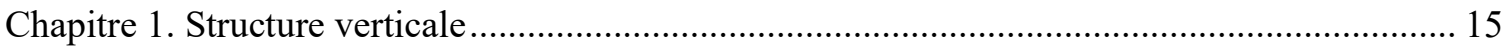

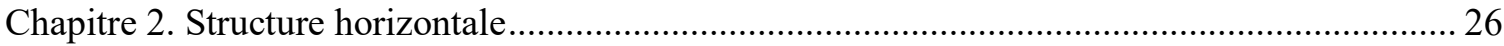

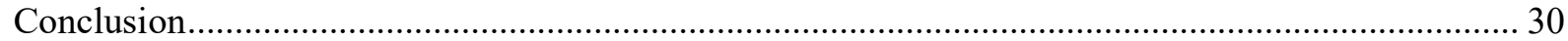




\section{Partie I. L'émergence de la concurrence fiscale passive}

En principe, les Etats membres n'ont pas transféré leurs compétences dans le domaine de la fiscalité directe à l'UE (cf. Titre 1). Cette retenue conduit à des formes de concurrence fiscale entre les Etats membres pour maintenir et /ou attirer des investissements et des activités sur leur territoire fiscal (cf. Titre 2).

\section{Titre 1. Souveraineté des Etats membres en matière de fiscalité directe}

Les Etats tirent leurs compétences dans le domaine de la fiscalité directe du concept de souveraineté (cf. Chapitre 1). Ces compétences n'ont pas été transférées à l'UE lors de l'accession des Etats membres (cf. Chapitre 2).

\section{Chapitre 1. Souveraineté fiscale et impôts directs}

La fiscalité est une émanation de la souveraineté de l'Etat ${ }^{1}$. Sa signification dans le domaine de la fiscalité est, en général, considérée comme une référence au pouvoir, à l'égard d'un territoire spécifique, de concevoir exclusivement un système fiscal et de l'appliquer'2. Ainsi, la souveraineté fiscale comporte généralement deux aspects : un pouvoir d'imposition objectif et un pouvoir d'imposition territorial ${ }^{3}$. Le pouvoir d'imposition objectif implique d'une part un pouvoir législatif de lever l'impôt (c'est-à-dire le pouvoir de décider des critères de fond et des règles de procédure pertinentes $)^{4} d$ 'autre part, un pouvoir d'exécution

\footnotetext{
${ }^{1}$ Il est généralement admis que le concept de souveraineté offre la justification légale du droit d'un Etat de taxer en vertu du droit international. (R.S.J. Martha, The jurisdiction to tax in international law, Deventer, Kluwer, 1989, 22). Alors que la souveraineté est un concept insaisissable, on considère généralement qu'elle renvoie à un ensemble de droits associés à un Etat (R.J. Jeffery, The impact of State sovereignty on global trade and international taxation, La Haye, Kluwer Law International, 1999, 26; J. Crawford, Brownlie's principles of public international law, Oxford University Press, 2012, 448). L'un des droits inclus dans le groupe de droits est la compétence territoriale, qui fait référence à la compétence d'un Etat pour réglementer la conduite des personnes associées au territoire physique de l'Etat State (R.S.J. Martha, op. cit., 13-15; J. Crawford, op. cit., 478). Le concept de compétence comporte de multiples facettes et comprend la compétence d'un Etat de légiférer (c.-à-d. la compétence normative) et de prendre des mesures exécutives ou judiciaires en vertu d'actes législatifs (c.-à-d. la compétence coercitive) pour les personnes ayant un lien identifiable avec le territoire d'un Etat State (voy. J. Crawford, op. cit., 478; M.N. Shaw, International law, Cambridge, Cambridge University Press, 2008, 645-646; R.J. Jeffery, op. cit., 26 et autres références).

${ }^{2}$ Voy. L. Cartou, Droit fiscal international et européen, Paris, Dalloz, 1981, 14-15 et A. Maitrot de la Motte, Droit fiscal de l'Union européenne, Bruxelles, Bruylant, 2016, 9.

${ }^{3}$ F. Vanistendael, General report on the fundamental freedoms and national sovereignty in the European Union, in F. Vanistendael (dir.), EU Freedoms and Taxation, Amsterdam, European Publications, 2005, 201, qui fait références à K. Tipke \& J. Lang, Steuerrecht, Ein systematischer Grundriss, Dr. Otto Schmidt Verlag, Cologne, 1996. Une distinction similaire est employée par E. Traversa et A. Pirlot, La souveraineté fiscale et la territorialité assiégée: dans quelle mesure les libertés de mouvement de l'UE devraient-elles avoir une incidence sur l'attribution territoriale des compétences fiscales entre les Etats membres?, in C. Brokelind (dir.), Principles of Law: Function, Status and Impact in EU Tax Law, Amsterdam, IBFD, 2014, pages en référence à A. Maitrot de la Motte, Souveraineté fiscale et construction communautaire. Recherche sur les impôts directs, Paris, LGDJ, 2005, 35-37 ; G. Tixier \& G. Gest, Droit fiscal international, Paris, PUF, 1990, 15 et L. Cartou, op. cit., 14-15.

${ }^{4}$ L. Cartou, op. cit., 14-15 et A. Maitrot de la Motte, Droit fiscal de l'Union européenne, Bruxelles, Bruylant, 2016, 9. Cela a été expliqué plus loin par Vanistendael comme suit: Le pouvoir législatif d'imposition peut également être subdivisé en plusieurs composantes: (1) le pouvoir de fixer le taux d'imposition, (2) le pouvoir de déterminer les règles de l'assiette fiscale, (3) le pouvoir de déterminer règles de procédure pour les audits, l'évaluation et les sanctions, (4) le pouvoir de réglementer les réclamations à l'égard des mesures fiscales et les procédures fiscales devant les tribunaux, le pouvoir d'organiser l'administration fiscale, (5) le pouvoir de négocier et de conclure des traités fiscaux. Le pouvoir de déterminer les règles de l'assiette à son tour, on peut subdiviser: (a) le pouvoir de fixer les règles structurelles essentielles de l'assiette (définition du bénéfice, de l'amortissement, des provisions, etc.), (b) le pouvoir de modifier le comportement des contribuables au moyens d'incitants (c) le
} 
pour appliquer les lois fiscales à un contribuable par les autorités (administratives ou judiciaires) compétentes ${ }^{5}$. Le pouvoir d'imposition territorial fait référence au pouvoir de décision sur le territoire sur lequel cet impôt est perçu ${ }^{6}$. Ces pouvoirs fiscaux s'exercent souverainement et de manière autonome, c'est-à-dire sans tenir compte des effets sur les systèmes d'impôts directs des autres Etats ${ }^{7}$. Par conséquent, les systèmes d'impôts directs des Etats du monde entier sont généralement non coordonnés et divergent considérablement.

\section{Chapitre 2. La compétence en matière de fiscalité directe n'a pas été transférée à l'UE}

Conformément à l'article 5, paragraphe 1, du TUE, les compétences de l'UE sont délimitées en vertu du principe d'attribution, ce qui signifie que «l'Union n'agit que dans les limites des compétences que les États membres lui ont attribuées dans les traités pour atteindre les objectifs que ces traités établissent» (article 5, paragraphe 2, du TUE). Par conséquent, " toute compétence non attribuée à l'Union dans les traités appartient aux États membres » (article 4, paragraphe 1 et article 5, paragraphe 2, du TUE).

Comme l'ont reconnu de nombreux auteurs, les dispositions des traités ne conférant pas à l'UE une compétence en matière des impôts directs, le domaine de la fiscalité directe semble relever de cette dernière catégorie des « compétences non attribuées à l'Union dans les traités » ou des « compétences retenues par les Etats membres ${ }^{8}$. Le professeur Lenaerts, par exemple, soutient que « [1] a fiscalité directe ne fait [...] l'objet, dans le traité, d'aucune attribution de compétence expresse à [l'UE]. C'est pourquoi la fiscalité directe relève, en principe, de la compétence des États membres ${ }^{9}$. De même, la jurisprudence de la CJUE ${ }^{10}$ indique que « la fiscalité directe relève de la compétence des États membres. Ainsi, ces derniers [...] sont seuls compétents pour concevoir des systèmes d'impôt sur les sociétés qu'ils considèrent les mieux adaptés aux besoins de leurs économies ${ }^{11}$.

pouvoir de déterminer les principes fondamentaux selon lesquels la loi fiscale sera appliquée (égalité, capacité de payer, règles sur l'évasion fiscale et les règles pénales en matière d'évasion fiscale) (F. Vanistendael, op. cit., 202). ${ }^{5}$ L. Cartou, op. cit., 15-16 et A. Maitrot de la Motte, Droit fiscal de l'Union européenne, Bruxelles, Bruylant, $2016,9$.

${ }^{6}$ E. Traversa et A. Pirlot, op. cit., 476.

${ }^{7}$ A.C. Dos Santos, L'Union européenne et la régulation de la concurrence fiscale, Bruxelles, Bruylant, 2009, 127 et H. Schaumburg, Steuerwettbewerb: Systematischer Überblick über die Gründe und die Auswirkungen des internationalen Steuerwettbewerbs, Internationale SteuerRundschau 2016, 372 et références citées. Voy. aussi pour comparaison L. Cartou, Droit fiscal international et européen, Paris, Dalloz, 1981, 15-16.

${ }^{8}$ A. Maitrot de la Motte, Droit fiscal de l'Union européenne, Bruxelles, Bruylant, 2016, 11. Voy. aussi infra.

${ }^{9} \mathrm{~K}$. Lenaerts et L. Bernardeau, L'encadrement communautaire de la fiscalité directe, CDE 2007, 29.

10 TPICE 18 déc. 2008, Government of Gibraltar et Royaume-Uni de Grande-Bretagne et d'Irlande du Nord c/ Commission, T-211/04 et T-215/04, Rec. II-03745, point 146 et références citées. Voir aussi CJUE 15 nov. 2011 , Commission et Royaume d'Espagne c/ Government of Gibraltar et Royaume-Uni de Grande-Bretagne et d'Irlande $d u$ Nord, C-106/09 P et C-107/09 P, Rec. I-11113, point 97 et CJUE 26 avr. 2018, ANGED, C-233/16, publié(e) au Recueil numérique (Recueil général), point 50.

${ }^{11}$ Cependant, cette position doit être qualifiée. Selon la CJUE, la fiscalité directe relève de la compétence des États membres « en l'état actuel du droit communautaire » (par exemple, CJCE 15 juil. 2004, Espagne c/ Commission, C-501/00, Rec. I-06717, point 123). En effet, on ne peut ignorer le fait que l'UE est attribué une compétence, en vertu de l'article 115, du TFUE, d'adopter des directives, qui peuvent avoir pour objet un rapprochement de la législation nationale sur les impôts directs. Nous partageons la conclusion de Peiffert : « l'essentiel de la matière de la fiscalité directe relève, en l'état du droit positif, de la compétence des Etats membres, du fait du non exercice par l'Union de sa compétence d'harmonisation ; c'est en cela qu'on peut parler de compétence nationale retenue » (O. Peiffert, A la recherche d'une exigence de concurrence fiscale 'loyale' en droit de l'Union européenne, Civitas Europa 2012, no. 2, 104 ; voyez également K. Lenaerts \& L. Bernardeau, op. cit., 31 ; L. Boucon, op. cit., 170 et L. Azoulai, La formule des compétences retenues des États membres devant la Cour de justice de l'Union européenne, in E. Neframi (dir.), Objectifs et compétences dans l'Union européenne, Bruxelles, Bruylant, 2013, 358). Cette observation a conduit certains auteurs à conclure que la fiscalité directe est même une compétence 
Le fait que les traités n'aient pas conféré directement à l'UE une compétence en matière de fiscalité directe ne signifie toutefois pas que les Etats membres ont reçu carte blanche pour agir librement dans ce domaine ${ }^{12}$. Comme nous le soulignons ci-dessous, les Etats membres doivent respecter le droit de l'UE dans l'exercice de leurs compétences en matière de la fiscalité directe, y compris le droit des aides d'Etat. Malgré cette limite, le constat demeure qu'en règle générale, les Etats membres ont conservé leurs compétences fiscales, avec pour résultat la coexistence au sein de l'UE de 28 systèmes fiscaux directs non coordonnés ${ }^{13}$.

\section{Titre 2. Concurrence fiscale entre les Etats membres}

La doctrine reconnait que l'absence de coordination des systèmes de fiscalité directe résultant de la souveraineté en matière de fiscalité directe des Etats membres conduit à la concurrence fiscale ${ }^{14}$. Celle-ci est une forme de concurrence réglementaire inter-juridictionnelle, fondée sur la prémisse que les Etats membres se font concurrence sur «le marché des droits fiscaux nationaux » pour conserver et attirer les investissements des entreprises ${ }^{15}$.

Cette concurrence se matérialise sous la forme d'un processus itératif de planification fiscale (agressive) ${ }^{16}$ par les entreprises et d'ajustement réglementaire par les Etats ${ }^{17}$. Pour être plus précis, la concurrence fiscale entre Etats exige (ou est facilitée) par la planification fiscale par les entreprises, et la planification fiscale par les entreprises nécessite (ou est facilitée par) la concurrence fiscale entre les Etats ${ }^{18}$. À cet égard, une distinction peut être effectuée entre deux formes de concurrence fiscale ${ }^{19}$, en fonction de la partie du processus itératif axée sur le rôle de l'Etat Membre concerné. L'accent peut être mis soit sur l'ajustement réglementaire de la part de l'Etat Membre, dans la mesure où il poursuit activement des stratégies de concurrence fiscale (cf. chapitre 1), soit sur les pratiques de planification fiscale des contribuables et sur la passivité des Etats membres à leur égard (cf. chapitre 2).

\section{Chapitre 1. Concurrence fiscale active}

En ce qui concerne le premier type de concurrence fiscale (active), l'accent est mis sur l'offre par un Etat membre d'un traitement fiscal avantageux au contribuable, à laquelle les contribuables réagissent en planifiant leurs activités en conséquence. En d'autres termes, les Etats membres peuvent conserver ou attirer des investissements sur leurs territoires (fiscaux)

partagée (par exemple, B. De Witte, Exclusive Member State Competences, in S. Garben \& I. Govaere (dir.), The Division of Competences between the EU and the Member States, Londres, Bloomsbury Publishing, 2017,61 ; P.J. Wattel, O. Marres \& H. Vermeulen (dir.), Terra/Wattel European Tax Law (Volume 1 - General Topics and Direct Taxation), Deventer, Kluwer, 2018, 12 et F. Vanistendael, Does the ECJ have the power of interpretation to build a tax system compatible with the fundamental freedoms ?, EC Tax Review 2008, 54)

${ }^{12}$ B. De Witte, Exclusive Member State Competences, S. Garben \& I. Govaere (ed.), The Division of Competences between the EU and the Member States, Londres, Bloomsbury Publishing, 2017, 60.

${ }^{13}$ D. Weber, In Search of a (New) Equilibrium Between Tax Sovereignty and the Freedom of Movement Within the EC, Intertax 2006, 586.

${ }^{14}$ A.C. Dos Santos, op. cit., 44-45. Voir aussi, C. Pinto, Tax competition and EU law, Amsterdam, Université d'Amsterdam, Thèse, 2002, 7.

${ }^{15}$ Comparer avec A. Saydé, Abuse of EU Law and Regulation of the Internal Market, Londres, Hart Publishing, 2014, 242. Dans la présente contribution, les autorités ne se pencheront pas sur les avantages et les inconvénients de la concurrence fiscale et des théories de l'intégration économique. Pour un examen détaillé, voyez par exemple, A. Saydé, op. cit, 227 et suiv. et A.C. Dos Santos, op. cit., 69 et suiv.

${ }^{16}$ Voy. infra (cf. chapitre 2) pour une définition de ce terme.

${ }^{17}$ A. Saydé, op. cit., 243.

${ }^{18}$ Comparez avec A.C. Dos Santos, op. cit, 34-35 et A. Saydé, op. cit., 243-244.

${ }^{19}$ Voir pour cette distinction, par analogie, A. Van den Bossche, Over de EU-grenzen aan fiscale concurrentie tussen lidstaten: interne markt, Unietrouw, staatssteun, SEW 2016, no. 7/8, 299-300. Les auteurs reconnaissent toutefois que cette distinction peut devenir floue dans certaines situations. 
(i) en introduisant ou appliquant des règles fiscales favorables dans une première étape (ii) qui déclenchent la planification fiscale (agressive) des contribuables qui s'installent sur le territoire dans un second temps.

Les exemples de pratiques de concurrence fiscale active sont multiples et concernent l'introduction d'incitations fiscales liées à certains investissements (par exemple dans certaines immobilisations) ou à certaines activités (activités de R\&D, activités de coordination, financements et holdings, etc.). Les auteurs de la présente contribution estiment que de tels régimes illustrent la conception originale du concept de concurrence fiscale, qui a fait l'objet d'un travail historique par $1^{\prime} \mathrm{OCDE}^{20}$ et par le groupe du Code de conduite (fiscalité des entreprises) de $1^{\prime} U^{21}$, qui a débuté à partir de la période 1997-1998 et a reçu de nouvelles impulsions ces dernières années dans le cadre de l'action 5 du projet de l'OCDE sur l'érosion de la base d'imposition et le transfert de bénéfices (BEPS ${ }^{22}$.

\section{Chapitre 2. Concurrence fiscale passive}

Dans le cadre du second type de concurrence fiscale (passive), l'accent est mis sur des pratiques de planification fiscale (agressive) de la part des contribuables, auxquelles un Etat membre ne réagit pas (de manière appropriée) en appliquant, par exemple, une règle défavorable au contribuable visant à neutraliser les avantages résultant d'une telle conduite (c'est-à-dire des règles anti-abus ou des règles sur les dispositifs hybrides). L'accent n'est donc pas placé sur l'offre active d'avantages fiscaux aux contribuables mais sur l'absence de réaction (appropriée) de l'Etat qui ne supprime pas les avantages fiscaux et financiers que les contribuables tirent des pratiques de planification fiscale agressive (PFA).

Ce concept de planification fiscale agressive «semble porter une connotation plus politiquement que juridiquement fructueuse $»^{23}$. Il fait généralement référence à des choix de lois indésirables, qui conduisent à une réduction artificielle des charges (fiscales) normalement supportées par une activité économique et qui sont donc susceptibles de modifier l'égalité des chances entre les entreprises. Tout en étant un concept insaisissable, la PFA est considérée comme un terme générique comprenant à la fois (i) l'évasion fiscale et (ii) l'arbitrage fiscal $^{24}$. Du point de vue de la fiscalité directe, les pratiques internationales d'évasion fiscale dans l'UE sont généralement perçues comme visant «à choisir un régime fiscal plus favorable et, partant, à éviter un régime moins favorable $»^{25}$. Les manifestations de pratiques d'évasion fiscale internationale sont multiples. Néanmoins, du point de vue de l'effet, de telles pratiques, qui se produisent généralement au sein du même groupe de sociétés, entraînent principalement (i) un transfert du revenu imposable ou du bénéfice vers un pays à faible taux d'imposition ou (ii) un transfert d'une dépense déductible vers un pays à forte imposition $^{26}$. L'arbitrage fiscal international n'est pas tant lié à un souhait d'éviter l'application

\footnotetext{
${ }^{20}$ OECD, Harmful Tax Competition - An Emerging Global Issue, Paris, OECD Publishing, 1998.

${ }^{21}$ Résolution du Conseil et des représentants des gouvernements des Etats membres, réunis au sein du Conseil, du 1er décembre 1997, sur un code de conduite en matière de fiscalité des entreprises, JOCE 1999 C 2, p. 2 et suiv.

${ }^{22}$ OECD, Countering Harmful Tax Practices More Effectively, Taking into Account Transparency and Substance, Action 5 - 2015 Final Report, OECD/G20 Base Erosion and Profit Shifting Project, Paris, OECD Publishing, 2015.

${ }^{23}$ P. Malherbe, Éléments de droit fiscal international, Bruxelles, Bruylant, 2015, 122, no. 214.

${ }^{24}$ Recommandation 2012/772/UE de la Commission du 6 décembre 2012 relative à la planification fiscale agressive, considérant $2:$ «La planification fiscale agressive consiste à tirer parti des subtilités d'un système fiscal ou des incohérences entre deux ou plusieurs systèmes fiscaux afin de réduire l'impôt à payer. Elle peut prendre de multiples formes. Parmi les conséquences de cette pratique, on peut citer les doubles déductions [...] et la double non-imposition $[\ldots] »$.

${ }^{25}$ A. Saydé, op. cit., 64 (traduction libre).

${ }^{26}$ Ibid.
} 
d'un système juridique afin d'être soumis à un autre système juridique, mais il fait référence à la situation dans laquelle « des contribuables qui sont assujettis à l'impôt dans plusieurs pays exploitent les différences entre les règles des régimes fiscaux [...] pour s'y conformer de manière technique dans les deux juridictions, tout en supportant une charge d'impôt nette totale moins élevée que si l'opération avait été soumise uniquement aux lois de l'une ou l'autre juridiction $»^{27}$. En d'autres termes, le contribuable profite des incohérences ou des conflits entre les règles fiscales des différents pays ${ }^{28}$.

Les manifestations de PFA par les contribuables ${ }^{29}$ conduisent sans aucun doute à une réduction artificielle des charges qui devraient en principe être supportées par les budgets des entreprises concernées. ${ }^{30}$ Autrement dit, de telles pratiques conduisent - en cas de succès - à une amélioration relative de la position fiscale des entreprises concernées, faussant ainsi les conditions de concurrence ${ }^{31}$. De ce point de vue, on pourrait donc soutenir que l'absence de réponse de la part de l'Etat à la PFA pratiquée par les contribuables peut constituer une forme de concurrence fiscale passive entre les Etats membres ${ }^{32}$.

\section{Partie II. L'utilisation du droit des aides d'Etat pour lutter contre la concurrence fiscale passive et son évaluation}

La Commission n'est pas restée inactive vis-à-vis des pratiques de concurrence fiscale entre les Etats membres puisqu'elle a utilisé ses prérogatives dans le cadre du contrôle des aides d'Etat en les appliquant à de telles pratiques dans la mesure où elles donnent lieu à des aides d'Etat au sens de l'article 107, paragraphe 1, TFUE ${ }^{33}$. Dès la fin des années 1990, la Commission a

\footnotetext{
27 A.H. Rosenzweig, Harnessing costs of international tax arbitrage, Virginia Tax Review 2007, no. 26, 555 (traduction libre).

${ }^{28}$ H.D. Rosenbloom, International Tax Arbitrage and the International Tax System, David R. Tillinghast Lecture on International Taxation, Tax Law Review 2000, no. 53/137, 142-143 et D.M. Ring, One nation among many: Policy implications of cross-border tax arbitrage, Boston College Law Review 2002, no. 44, 79.

${ }^{29}$ D'une certaine manière, la planification fiscale agressive peut également être considérée comme une forme de concurrence entre les entreprises pour réduire leur fardeau fiscal vis-à-vis des concurrents et ainsi améliorer leur position concurrentielle. Toutefois, ce n'est pas la même chose que la concurrence fiscale au sens interjuridictionnel, voy. A.C. Dos Santos, op. cit., 32-34.

${ }^{30}$ Comparez avec A.C. Dos Santos, op. cit., 32-33, et références citées.

${ }^{31}$ Ibid.

${ }^{32}$ Comparez avec P. Malherbe, Éléments de droit fiscal international, Bruxelles, Bruylant, 2015, 122 (« Les règles anti-abus sont donc des composantes cruciales de la compétitivité internationale et de l'attrait d'un système d'impôt ») ; A.C. Dos Santos, op. cit., 38-39, qui déclare qu'Aujean (M. Aujean, Los sistemas fiscales en la Unión Europea: competencia o coordinación, Hacienda Pública Española 1997) discute de plusieurs stratégies de planification fiscale par lesquelles les contribuables / entreprises peuvent tirer certains avantages, puis juge que « celles-ci n'impliquent aucun comportement de l'Etat, aucun bonus offert par lui. Même si elles peuvent donner origine à des formes dommageables de concurrence, ces formes sont d'une autre nature (concurrence fiscale implicite ou passive) ». Nous notons que cette forme de concurrence fiscale passive est différente de la concurrence fiscale passive mentionnée par Pinto (C. Pinto, op. cit., 14) à savoir « promulguer des contre-mesures unilatérales ciblant spécifiquement les mesures des pays activement engagés dans la concurrence fiscale » (traduction libre).

${ }^{33}$ C'est-à-dire répondre à tous les éléments constitutifs d'une aide d'Etat telles qu'ils sont définis dans cette disposition. Les auteurs de la présente contribution concluent qu'il n'y a pas de lien de causalité entre l'existence d'une concurrence fiscale (passive ou active) entre les Etats membres, d'une part, et l'application du système de contrôle des aides d'Etat, d'autre part. Voy. également la Résolution du Conseil et des représentants des gouvernements des Etats membres, réunis au sein du Conseil, du 1er décembre 1997, sur un code de conduite en matière de fiscalité des entreprises, JOCE 1999 C 2, p. 2 et suiv., point 30 (« La qualification de mesure fiscale dommageable au titre du code de conduite n'affecte pas la qualification éventuelle de la mesure en tant qu'aide d'état. ») et A. Maitrot de la Motte, La concurrence fiscale dans la jurisprudence de la Cour de justice, in E. Mazuyer, E. Carpano et M. Chastagnaret (dir.), La concurrence réglementaire, sociale et fiscale dans l'Union européenne, Bruxelles, Larcier, 2016, 295, en référence aux conclusions de l'av. gén. dans C-106/09 P et C-107/09 $\mathrm{P}$, Commission et Royaume d'Espagne c/ Government of Gibraltar et Royaume-Uni de Grande-Bretagne et d'Irlande du Nord, points 122-135 et R. Luja, General report, in JL da Cruz Vilaça et al. (dir.), Taxation, State aid
} 
commencé à examiner diverses pratiques actives de concurrence fiscale des Etats membres (principalement des avantages fiscaux accordés aux centres de coordination) et a constaté que de tels régimes comportaient l'octroi d'aides d'Etat ${ }^{34}$. Cette analyse a été confirmée par la CJUE ${ }^{35}$. Durant ces dernières années, la Commission a étendu le champ d'application du contrôle des aides d'Etat à l'examen de formes passives de concurrence

and distortions of competition, Conference proceedings XXVIII FIDE Congress, Coimbra, Almedina, 2018, 55). En d'autres termes, ce n'est pas parce qu'une certaine forme de comportement est qualifiée de concurrence fiscale qu'un tel comportement est automatiquement pertinent du point de vue des aides d'Etat (voy. par exemple une approche différente par E. Meier \& Th. Perrot, Les aides d'État comme instrument de lutte contre la concurrence fiscale dommageable: la pierre philosophale?, Revue de Droit Fiscal 2002, 137, qui ont soutenu qu'il existe un lien formel entre pratiques fiscales dommageables et aides d'Etat). Cela peut s'expliquer par le fait que la lutte contre la concurrence fiscale et les règles sur les aides d'Etat ont une orientation différente : si la concurrence fiscale concerne la concurrence entre Etats membres, le droit des aides d'Etat concerne les distorsions de concurrence entre entreprises (voyez par exemple J. Biancarelli, Le contrôle de la justice des communautés européennes dans les aides publiques, AJDA 1993, 412, citant J.-Y. Chérot, La discipline des nations dans la communauté européenne, Revue d'économie industrielle 1993, 222: « La concurrence dans le marché commun est une concurrence entre entreprises et non une concurrence entre Etats »). Néanmoins, il existe de facto un lien entre la concurrence fiscale et les distorsions de concurrence entre entreprises.

${ }^{34}$ Décision 2002/937/CE de la Commission du 10 juillet 2002 relative à l'aide d'État accordée par la Finlande en faveur des compagnies d'assurance captives de la région d'Åland, JOCE 2002 L 329, p. 22-29; Décision 2003/81/CE de la Commission du 22 août 2002 relative au régime d'aides d'État que le Royaume d'Espagne applique aux 'centres de coordination de Biscaye', JOUE 2003 L 31, p. 26-31; Décision 2003/512/CE de la Commission du 5 septembre 2002 relative au régime d'aide exécuté par l'Allemagne en faveur des centres de contrôle et de coordination, JOUE 2003 L 177, p. 17-21; Décision 2003/438/CE de la Commission du 16 octobre 2002 concernant le régime d'aide d'État (Sociétés de financement) mis à exécution par le Luxembourg, JOUE 2203 L 153, p. 40-48; Décision 2003/501/CE de la Commission du 16 octobre 2002 concernant le régime d'aide d'État (Centres de coordination) mis à exécution par le Luxembourg, JOUE 2003 L 170, p. 20-28; Décision 2003/230/CE de la Commission du 11 décembre 2002 relative au régime d'aide existant que l'Italie avait été autorisée à exécuter en faveur du centre de services financiers et d'assurance de Trieste, JOUE 2003 L 91, p. 4750; Décision 2003/883/CE de la Commission du 11 décembre 2002 concernant le régime d'aide d'État (Centrales de trésorerie) mis à exécution par la France, JOUE 2003 L 330, p. 23-29; Décision 2003/515/CE de la Commission du 17 février 2003 concernant le régime d'aide mis à exécution par les Pays-Bas pour les activités de financement internationales, JOUE 2003 L 180, p. 52-66; Décision 2003/755/CE de la Commission du 17 février 2003 concernant le régime d'aides mis en œuvre par la Belgique en faveur des centres de coordination établis en Belgique, JOUE 2003 L 282, p. 25-45; Décision 2003/601/CE de la Commission du 17 février 2003 concernant le régime d'aide d'Irlande (revenus étrangers), JOUE 2003 L 204, p. 51-59; Décision 2004/76/CE de la Commission du 13 mai 2003 concernant le régime d'aide d'État mis à exécution par la France en faveur des quartiers généraux et centres de logistique, JOUE 2004 L 23, 1-13; Décision 2004/77/CE de la Commission du 24 juin 2003 concernant le régime d'aides mis à exécution par la Belgique sous la forme d'un régime fiscal de ruling applicable aux US Foreign Sales Corporations, JOUE 2004 L 23, p. 14-26; Décision 2005/77/CE de la Commission du 30 mars 2004 relative au régime d'aides mis à exécution par le Royaume-Uni en faveur des «qualifying companies» de Gibraltar, JOUE 2005 L 29, p. 24-38; Décision 2005/261/CE de la Commission du 30 mars 2004 relative au régime d'aides que le Royaume-Uni envisage de mettre à exécution concernant la réforme de l'impôt sur les sociétés par le gouvernement de Gibraltar, JOUE 2005 L 85, p. 1-26 et Décision 2006/940/CE de la Commission du 19 juillet 2006 concernant le régime d'aide C 3/2006 mis en œuvre par le Luxembourg en faveur des sociétés holdings 1929 et des holdings milliardaires, JOUE 2006 L 366, p. 47-61.

${ }^{35}$ CJCE 22 juin 2006, Belgique et Forum 187 ASBL c/ Commission, C-182/03 et C-217/03, Rec. I-05479. 
fiscale $^{36}$ (cf. titre 1). Les procédures visant ces pratiques passives soulèvent de nombreuses questions qui appellent une évaluation institutionnelle (cf. titre 2$)^{37}$.

\section{Titre 1. Utilisation du droit des aides d'Etat pour lutter contre la concurrence fiscale passive}

A partir de 2013, une nouvelle ère de contrôle des aides d'Etat semble avoir commencé. En effet, la Commission a ouvert des procédures analysant diverses pratiques qui nous semblent pouvoir être assimilées à des pratiques passives de concurrence fiscale où les Etats ne réagissent pas (de manière appropriée) aux pratiques de PFA des entreprises. Une telle position (trop) permissive envers les contribuables peut être adoptée dans des cas individuels ou intégrée dans une politique plus large.

La Commission prend des mesures en ce qui concerne les deux types de situations. A ce jour, elle a examiné les octrois de rescrits fiscaux par les Etats Membres ${ }^{38}$ et a spécifiquement ouvert des procédures contre les rescrits ${ }^{39}$ accordés par les Pays-Bas à Starbucks ${ }^{40}$ et à IKEA ${ }^{41}$, au Luxembourg à Amazon ${ }^{42}$, à Fiat ${ }^{43}$, à McDonald's ${ }^{44}$ et à GDF Suez (Engie ${ }^{45}$ et par 1'Irlande à $\mathrm{Apple}^{46}$. En outre, elle a également examiné les régimes d'aides par lesquels une position (trop)

\footnotetext{
${ }^{36}$ Ces procédures ont été considérées par la Commission comme une montée en puissance de sa politique en matière d'examen dans le domaine de la fiscalité directe (P. Lowe, The Commission's powers to enforce the law: And to propose changes to it, EL Rev. 2017, 911). Cependant, les auteurs estiment qu'il convient de distinguer ces enquêtes des précédentes dans la mesure où ce qui est critiqué par la Commission n'est pas l'introduction active de lois ou de règlements fiscaux bénéfiques, mais l'attitude persistante de certains Etats membres vis-à-vis de la PFA (qui est une forme de concurrence fiscale passive). Voir pour d'autres raisons pourquoi l'approche de la Commission est nouvelle, P. Lowe, op. cit., 911.

${ }^{37}$ Notez que l'un des auteurs de cette contribution prépare actuellement une thèse de doctorat liée à plusieurs de ces questions. La présente contribution ne préjuge en rien de l'analyse faite dans cette thèse.

${ }^{38}$ Voy. le document de travail de la DG Concurrence sur les aides d'Etat et les rescripts fiscaux ( Working Paper on State Aid and Tax Rulings), http://ec.europa.eu.

${ }^{39}$ Egalement appelés « rulings » ou « tax rulings » en langue anglaise.

${ }^{40}$ Décision (UE) 2017/502 de la Commission du 21 oct. 2015 concernant l'aide d'État SA.38374 (2014/C ex 2014/NN) mise à exécution par les Pays-Bas en faveur de Starbucks, JOUE 2017 L 83, p. 38-115. Cette décision est contestée par les Pays-Bas et Starbucks devant le Tribunal (affaires T-760/15 et T-636/16, respectivement).

${ }^{41}$ Commission européenne, 18 déc. 2017, Possible aide d'État en faveur d'Inter IKEA, Invitation à présenter des observations en application de l'article 108, paragraphe 2, du traité sur le fonctionnement de l'Union européenne, 2018/C 121/04, JOUE 2018 C 121, p. 30-86. Cette décision n'est qu'une décision d'ouverture, c'est-à-dire que la procédure d'enquête formelle est toujours pendante à la date de finalisation de cette contribution.

${ }^{42}$ Décision (UE) 2018/859 de la Commission du 4 oct. 2017 concernant l'aide d'État SA.38944 (2014/C) (ex 2014/NN) mise à exécution par le Luxembourg en faveur d'Amazon, JOUE 2018 L 153, p. 1-142. Cette décision est contestée par le Luxembourg et Amazon devant le Tribunal (affaires T-816/17 et T-318/18, respectivement).

${ }^{43}$ Décision (UE) 2016/2326 de la Commission du 21 oct. 2015 concernant l'aide d'État SA.38375 (2014/C ex 2014/NN) mise à exécution par le Luxembourg en faveur de Fiat, JOUE 2016 L 351, p. 1-67. Cette décision est contestée par le Luxembourg et Fiat devant le Tribunal (affaires T-755/15 et T-759/15, respectivement).

${ }^{44}$ Commission européenne, 18 déc. 2017, Aide présumée en faveur de McDonald's, Invitation à présenter des observations en application de l'article 108, paragraphe 2, du traité sur le fonctionnement de l'Union européenne, 2016/C 258/03, JOUE 2016 C 258, p. 11-48 (décision d'ouverture de la procédure formelle). La procédure formelle d'examen a été clôturée et la décision de la Commission a été rendue le 19 septembre 2018 mais la version publique n'était pas accessible le à la date de finalisation de cette contribution. Voy. Commission européenne, Aides d'État: l'enquête de la Commission n'a pas conclu que le Luxembourg avait accordé un traitement fiscal sélectif à McDonald's, 19 septembre 2018, IP/18/5831.

${ }^{45}$ Décision (UE) de la Commission du 20 juin 2018 concernant l'aide d'Etat SA.44888 (NN / 2016) (ex EO / 2016) mise à exécution par le Luxembourg en faveur de GDF Suez, non encore publiée en JOUE. Cette décision est contestée par le Luxembourg et ENGIE devant le Tribunal (affaires T-516/18 et T-525/18, respectivement).

${ }^{46}$ Décision (UE) 2017/1283 de la Commission du 30 août 2016 concernant l'aide d'État SA.38373 (2014/C) (ex 2014/NN) (ex 2014/CP) octroyée par l'Irlande en faveur d'Apple, JOUE 2017 L 187, p. 1-110. Cette décision est contestée par l'Irlande et Apple devant le Tribunal (affaires T-778/16 et T-892/16, respectivement).
} 
passive a été prise vis-à-vis d'un groupe plus large de contribuables, comme dans le cadre du régime belge des bénéfices excédentaires ${ }^{47}$ et l'affaire anglaise des $\mathrm{CFC}^{48}$. L'essentiel de ces procédures sera très succinctement traité dans les sections suivantes, selon qu'il s'agit de la réaction des Etats membres à (i) l'évasion fiscale internationale ${ }^{49}$ (cf. chapitre 1) ou (ii) à l'arbitrage fiscal international (cf. chapitre 2).

\section{Chapitre 1. Évasion fiscale internationale}

Les procédures entamées par la Commission en raison de l'attitude des Etats membres vis-àvis de l'évasion fiscale internationale pratiquée par les entreprises impliquent en grande partie des questions fiscales liées au traitement des sociétés faisant partie d'un groupe multinational qui transfèrent les bénéfices de la juridiction d'un Etat membre vers des juridictions présentant un taux d'imposition effectif inférieur. Ce transfert de bénéfices est possible parce que tous les Etats membres reconnaissent et acceptent - à des fins fiscales - la personnalité juridique distincte des entités du groupe. Cela signifie que chaque entité du groupe est, en principe, soumise à l'impôt sur les sociétés sur sa propre base taxable au taux de la juridiction dans laquelle elle est résidente d'un point de vue fiscal ${ }^{50}$. La comptabilisation à des fins fiscales des transactions entre membres d'un même groupe comporte évidemment le risque que les entités du groupe n'établissent pas des relations contractuelles reflétant les conditions du marché et transfèrent ainsi leurs bénéfices vers des environnements à fiscalité plus faible. Cette évasion fiscale internationale peut s'effectuer de plusieurs façons.

Premièrement, les sociétés du groupe pourraient utiliser le mécanisme des prix de transfert, permettant de fixer un prix à un niveau artificiellement soit élevé soit faible pour la livraison de biens ou la fourniture de services entre eux. Ce mécanisme permet de réduire la base imposable pour la société du groupe située dans une juridiction où le niveau de taxation est important. À cet égard, la Commission allègue dans nombre de ses procédures qu'un Etat membre octroie une aide d'Etat lorsqu'il accepte (c'est-à-dire ne corrige pas) des prix pratiqués entre les sociétés du même groupe qui ne sont pas conformes aux conditions du marché et qui réduisent la charge fiscale pour une société dans l'Etat membre concerné. Le raisonnement à l'appui de cette conclusion suit en grande partie le même schéma dans toutes ces procédures ${ }^{51}$. Sans entrer dans les détails, le cœur de l'argument de la Commission réside dans son interprétation de l'article 107, paragraphe 1, TFUE comme incorporant un principe général d'égalité de traitement qui exige que les sociétés du groupe et les sociétés indépendantes soient traitées de la même façon. Pour être plus précis, les Etats membres devraient veiller à ce qu'une société d'un groupe « ne bénéficie pas d'un traitement plus favorable, en application du système général de l'impôt sur les sociétés, que celui réservé à des sociétés non intégrées dont les bénéfices imposables

\footnotetext{
${ }^{47}$ Décision (UE) 2016/1699 de la Commission du 11 janv. 2016 relative au régime d'aides d'État concernant l'exonération des bénéfices excédentaires SA.37667 (2015/C) (ex 2015/NN) mis en œuvre par la Belgique, JOUE 2016 L 260, p. 61-103. Cette décision est contestée par la Belgique et plusieurs bénéficiaires devant le Tribunal (affaires T-131/16 et au moins 28 affaires introduites par des bénéficiaires).

${ }^{48}$ Commission européenne, 26 oct. 2017, Régime d'aides d'État potentiel concernant l'exonération sur le financement des groupes instaurée par le Royaume-Uni, Invitation à présenter des observations en application de 1'article 108, paragraphe 2, du traité sur le fonctionnement de l'Union européenne, 2017/C 400/03, JOUE 2017 C 400, p. 10-31. Cette décision n'est qu'une décision d'ouverture, c'est-à-dire que la procédure d'enquête formelle est toujours pendante à la date de finalisation de cette contribution.

${ }^{49}$ Les auteurs ont choisi de ne pas aborder le cas d'opérations d'évasion fiscale purement nationales, qui est en cause dans l'affaire GDF Suez/Engie.

${ }^{50}$ Comparez avec Y. Brauner, Transfer Pricing and Tax Avoidance, in A.P. Dourado, Tax Avoidance Revisited in a BEPS Context, Amsterdam, IBFD, 2017, 63-64.

${ }^{51}$ Voir pour un résumé utile des décisions de la Commission, J. Rapp \& R. Ianus, Institutional report, in J.L. da Cruz Vilaça et al. (dir.), Taxation, State aid and distortions of competition, Conference proceedings XXVIII FIDE Congress, Coimbra, Almedina, 2018, 124 et suiv.
} 
sont déterminés par le marché $»^{52}$. Par conséquent, les Etats membres devraient appliquer un " principe de pleine concurrence ${ }^{53}$ selon lequel les transactions intragroupes devraient être tarifées comme si elles avaient été convenues entre des sociétés indépendantes négociant dans des circonstances comparables ${ }^{54}$. Selon la Commission, c'est parce que ce principe de pleine concurrence est une déclinaison spécifique du principe d'égalité consacré à l'article 107, paragraphe 1, du TFUE ${ }^{55}$, qu'il «fait [...] nécessairement partie intégrante de l'appréciation par la Commission [...] des mesures fiscales accordées aux sociétés d'un groupe, indépendamment de la question de savoir si un État membre a incorporé ce principe dans son système juridique national $\|^{56}$. Par conséquent, il semble que la Commission ne tienne pas compte des règles qui sont présentes dans l'ordre juridique national et qu'elle impose en fait son propre principe de référence pour évaluer si une aide est accordée ou non. Deux situations sont possibles. Dans la première, des principes de libre concurrence (nationaux) sont ancrés dans l'ordre juridique national comme c'est le cas pour de nombreux Etats membres ${ }^{57}$. Dans ce cas, l'argumentation de la Commission présente une portée incertaine : soit elle soutient que le problème se situe au niveau de la législation et ensuite la question est de savoir si une telle législation est suffisamment efficace ou non; soit la Commission admet que chaque Etat membre dispose de ses propres règles pour lutter contre les stratégies reposant sur le mécanisme des prix de transfert, mais que les Etats membres concernés ont mal appliqué ces règles dans les cas spécifiques faisant l'objet de l'examen. Dans le second type de situation où les Etats membres ne disposent pas de règles incluant un principe de libre concurrence dans leur ordre

\footnotetext{
${ }^{52}$ Décision Fiat, point 228; décision Starbucks, point 264 et décision Apple, point 256.

${ }^{53}$ Egalement appelé « arm's length principle » en langue anglaise.

${ }^{54}$ Décision Amazon, point 402. Voir également la décision Fiat, points 224-225; décision Apple, point 251; Starbucks décision, point 261 et décision IKEA, point 111. Voir aussi J. Rapp \& R. Ianus, op. cit., 125.

${ }^{55}$ Comparer la décision Fiat, point 228 et la décision Starbucks, point 264: « le principe de pleine concurrence que la Commission applique dans le cadre de son appréciation des aides [...] repose sur un principe général d'égalité de traitement en matière de taxation qui relève de l'application de l'article 107, paragraphe 1, du TFUE ».

${ }^{56}$ Décision Fiat, point 228 et décision Starbucks, point 264. Voir aussi. J. Rapp \& R. Ianus, op. cit., 132-133, qui soutiennent en outre qu'il ne s'agit pas d'un principe de pleine concurrence mais d'un principe universel qui est inhérent à tout système fiscal national (ce qui soulève la question de savoir s'il s'agit d'une sorte de principe de droit international coutumier).

${ }^{57}$ Par exemple, l'Allemagne ( 1 Abs. 3 AStG, voyez R. Wernsmann \& P. Wagner, « Germany » in J.L. da Cruz Vilaça et al. (dir.), Taxation, State aid and distortions of competition, Conference proceedings XXVIII FIDE Congress, Coimbra, Almedina, 2018, 315-316); l'Autriche ( 66 Z 6 lit. a EStG; voyez M. Schwaiger \& I. Kerschner, "Austria », in J.L. da Cruz Vilaça et al. (dir.), Taxation, State aid and distortions of competition, Conference proceedings XXVIII FIDE Congress, Coimbra, Almedina, 2018, 173-174), la Belgique (l'article 26, 79, 185 et 207 du CIR); la France (l'article 57 du CGI ; voyez S. De La Rosa et al., « France » in J.L. da Cruz Vilaça et al. (dir.), Taxation, State aid and distortions of competition, Conference proceedings XXVIII FIDE Congress, Coimbra, Almedina, 2018, 284-285); l'Espagne (l'article 18 du Ley del Impuesto sobre Sociedades et l'article $15 \mathrm{du}$ Ley del Impuesto sobre la Renta de no Residentes, Real Decreto Legislativo 5/2004; voyez A. Martin-Jiménez \& J.J. Piernas-López, «Spain » in J.L. da Cruz Vilaça et al. (dir.), Taxation, State aid and distortions of competition, Conference proceedings XXVIII FIDE Congress, Coimbra, Almedina, 2018, 501-502) ;

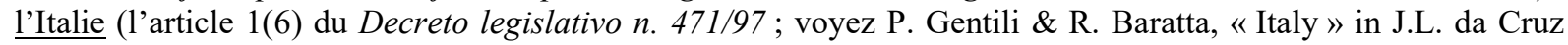
Vilaça et al. (dir.), Taxation, State aid and distortions of competition, Conference proceedings XXVIII FIDE Congress, Coimbra, Almedina, 2018, 404-405); les Pays-Bas (l'article 3.8 du Wet inkomstenbelasting 2001; voyez S. Douma, « the Netherlands » in J.L. da Cruz Vilaça et al. (dir.), Taxation, State aid and distortions of competition, Conference proceedings XXVIII FIDE Congress, Coimbra, Almedina, 2018, 532) et le Royaume-Uni (Schedule 28AA du ICTA 1988 et, maintenant, Section 164 du TIOPA 2010; voyez A. Biondi \& K. Stricklin-Coutinho, «United Kingdom» in J.L. da Cruz Vilaça et al. (dir.), Taxation, State aid and distortions of competition, Conference proceedings XXVIII FIDE Congress, Coimbra, Almedina, 2018, 558-559). L'Irlande n'a introduit de règles relatives aux prix de transfert qu'en 2010 (Part 35A du TCA ; voyez G. Duggan \& C. Toland, «Ireland » in J.L. da Cruz Vilaça et al. (dir.), Taxation, State aid and distortions of competition, Conference proceedings XXVIII FIDE Congress, Coimbra, Almedina, 2018, 384).
} 
juridique national, ces Etats membres se verraient néanmoins obligés d'appliquer ces règles et accepter que leur non-respect entraîne l'octroi d'une aide d'Etat.

Une seconde possibilité d'évasion fiscale internationale consiste pour les sociétés d'un groupe à transférer des activités ou des actifs à une société étrangère contrôlée (en anglais « Controlled Foreign Companies » ou " CFC ») établie dans une juridiction à fiscalité réduite, au sein de laquelle le revenu s'accumule et reste ainsi non imposé au niveau de la société du groupe de contrôle $^{58}$. Pour éliminer ces pratiques, de nombreux Etats membres - comme le Royaume-Uni - ont adopté une législation sur les CFC qui attribue et taxe une part proportionnelle du revenu non distribué qui a été artificiellement détourné de la société mère (britannique) vers sa filiale $^{59}$. Cependant, la législation du Royaume-Uni sur les CFC prévoit une exception spécifique à cette règle d'attribution ( «l'exemption de financement de groupe ») qui permet à certains bénéfices financiers de la CFC d'être exemptés de l'attribution et de la taxation auprès de la société mère britannique ${ }^{60}$. Selon la Commission, l'exemption de financement de groupe implique que « certaines sociétés résidentes du Royaume-Uni ne sont pas imposées sur certains bénéfices financiers artificiellement détournés gagnés par une CFC qu'ils contrôlent tandis que d'autres bénéfices artificiellement détournés de CFC, contrôlés par d'autres sociétés résidentes du Royaume-Uni dans une situation juridique et factuelle comparable, ont été imposées $»^{61}$. En d'autres termes, l'exception implique que le régime est sélectif et donc qu'il y a une aide d'Etat.

En somme, il semble que la Commission avance quatre types d'arguments dans ses procédures concernant l'attitude des Etats membres vis-à-vis des pratiques d'évasion fiscale, à savoir que l'octroi d'un avantage sélectif peut résulter (i) de l'absence d'une règle anti-abus dans la législation nationale, (ii) de la définition de la règle anti-abus d'une manière qui ne s'applique pas (avec la même efficacité) aux contribuables qui sont dans des situations comparables, (iii) de la création d'une exception législative à une règle anti-abus ou (iv) d'une mauvaise application d'une règle nationale anti-abus à un contribuable ${ }^{62}$.

\section{Chapitre 2. Arbitrage fiscal international}

Les procédures de la Commission présentant un aspect relatif à l'arbitrage fiscal international, telles que l'affaire McDonald's et l'affaire belge sur le régime d'exonération des bénéfices excédentaires, sont très complexes et une analyse détaillée sortirait du cadre de cette contribution. Nous nous limitons à en décrire les éléments pertinents pour cette contribution.

\footnotetext{
${ }^{58} \mathrm{Il}$ en résulte un report d'impôt dans l'Etat dans lequel la société mère est un résident à des fins fiscales. Voir par exemple L. De Broe, International Tax Planning and Prevention of Abuse. A Study under Domestic Tax Law, Tax Treaties and EC Law in relation to Conduit and Base Companies, Amsterdam, IBFD, 2008, sec. 2.1.3; B.J. Arnold \& P. Dibout, General Report, in IFA, Limits on the Use of Low-Tax Regimes by Multinational Businesses: Current Measures and Emerging Trends, Cah. Dr. Fisc. Int., Vol. LXXXVIb, The Hague, Kluwer International, 2001, 3839.

${ }^{59}$ Décision du Royaume-Uni sur les règles régissant les CFC, points 5 et 61 . Voir aussi le communiqué de presse (IP/17/4201): "Les règles britanniques sur les sociétés étrangères contrôlées (SEC) ont pour objectif général d'empêcher les sociétés du Royaume-Uni d'utiliser une filiale située dans un pays à la fiscalité faible ou nulle pour éluder l'impôt au Royaume-Uni. Elles permettent notamment à l'administration fiscale britannique de réaffecter à la société mère britannique tous les bénéfices transférés artificiellement vers une filiale offshore et donc de les taxer en conséquence. ».

${ }^{60}$ En l'absence du « Group Financing Exemption », le revenu de financement serait attribué (et imposé entre les mains de) la société mère britannique.

${ }^{61}$ Décision du Royaume-Uni sur les règles régissant les CFC, point 62; voir aussi point 71 (traduction libre).

${ }^{62}$ L'argument selon lequel une mauvaise application d'une règle anti-abus nationale (ou son absence) pourrait impliquer l'octroi d'une aide d'État est également présenté dans l'affaire GDF Suez/Engie (voir points 289 à 312), que nous avons choisi de ne pas examiner dans cette contribution (voir note 49).
} 
La groupe McDonald's dispose d'une filiale qui est résidente fiscale luxembourgeoise ('McDonald's Luxembourg') et à laquelle un certain nombre de droits de franchise a été octroyé. McDonald's Luxembourg, qui détient une succursale aux Etats-Unis, a ensuite attribué ces droits en interne à la succursale américaine, via laquelle elle perçoit des redevances de franchisés. McDonald's Luxembourg est, en principe, soumise à la juridiction fiscale du Luxembourg (l'Etat d'origine) et des Etats-Unis (l'Etat hôte). Pour remédier à la double imposition, la Convention visant à éviter les doubles impositions (CDI) entre le Luxembourg et les Etats-Unis prévoit que (i) les Etats-Unis peuvent imposer les bénéfices de la société luxembourgeoise, dans la mesure où cette dernière a un établissement stable aux Etats-Unis ${ }^{63}$ et (ii) le Luxembourg doit exempter les revenus qui "sont imposables" aux EtatsUnis $^{64}$. Conformément au droit fiscal international ${ }^{65}$, l'expression « sont imposables » implique que même si les Etats-Unis n'imposent pas, en droit interne, les revenus de l'établissement stable de la société luxembourgeoise, le Luxembourg doit exempter le revenu, et c'est précisément le cas dans cette affaire ${ }^{66}$. Deux décisions fiscales anticipatives ont été octroyées par l'administration luxembourgeoise à McDonald's Luxembourg, qui confirment ce traitement fiscal. Cette double non-imposition a été critiquée dans un premier temps par la Commission, en ce sens qu'elle «ne semble respecter ni la formulation de ses dispositions [de la CDI], ni leur objectif $»^{67}$. En effet, la Commission a prétendu, en substance, dans sa décision d'ouverture de la procédure formelle que les autorités fiscales luxembourgeoises ont mal appliqué la $\mathrm{CDI}^{68}$ en exemptant les bénéfices qui n'auraient jamais pu être imposés aux Etats-Unis, ce qui conduit à un avantage sélectif ${ }^{69}$. Cependant, en concluant la procédure formelle, la Commission considère qu'elle n'a pu établir que « l'interprétation de la [CDI] donnée par la [...] décision fiscale anticipative était incorrecte, même si elle a entraîné la double non-imposition des redevances attribuées à la succursale américaine. En conséquence, [...] les autorités luxembourgeoises n'ont pas fait une application erronée de la [CDI et] l'avantage fiscal accordé [...] ne peut pas être considéré comme une aide d'État $»^{70}$.

Dans l'affaire belge sur le régime d'exonération des bénéfices excédentaires, la Belgique avait octroyé une réduction de la base imposable d'une société belge faisant partie d'un groupe multinational. La Commission soutenait que la réduction de la base imposable était prima facie sélective, soit parce qu'elle constituait une mauvaise application du principe de pleine concurrence (voir ci-dessus), soit parce qu'en exonérant de l'impôt sur les sociétés une partie de leur bénéfice réellement enregistré, elle dérogeait au système commun d'imposition des bénéfices des sociétés qui est fondé sur le principe d'imposition du bénéfice total. La Commission faisait également valoir qu'un tel avantage prima facie sélectif ne pouvait être justifiée, comme la Belgique le prétendait, par la nature et l'économie générale du système fiscal belge car la justification de la double non-imposition ne peut être invoquée qu'en présence

\footnotetext{
${ }^{63}$ Article 7, paragraphe 1, de la CDI.

${ }^{64}$ Article 25, paragraphe 2, a), de la CDI.

${ }^{65}$ Voy. OCDE, Modèle de Convention fiscale concernant le revenu et la fortune 2014, Éditions OCDE, Paris, Introduction, point 25.1 .

${ }^{66}$ Cette affaire est un plus complexe, mais ses détails sortent du cadre de cette contribution.

${ }^{67}$ Décision McDonald's, point 86.

${ }^{68}$ Décision McDonald's, point 91: « la confirmation de l'administration fiscale luxembourgeoise que les revenus pouvaient être exemptés de l'impôt luxembourgeois sur le revenu des collectivités en vertu de l'article 25 de la CDI Luxembourg - États-Unis repose sur une mauvaise application de cette disposition ».

${ }^{69}$ Décision McDonald's, points 91-92.

${ }^{70}$ Commission européenne, Aides d'État: l'enquête de la Commission n'a pas conclu que le Luxembourg avait accordé un traitement fiscal sélectif à McDonald's, 19 septembre 2018, IP/18/5831. Nous notons également que dans son communiqué de presse, la Commission fait état d'une législation luxembourgeoise en projet au 19 juin 2018 qui vise à modifier ses règles fiscales pour s'aligner sur les principes du BEPS de l'OCDE et ainsi éviter une situation semblable de double non-imposition.
} 
d'une double imposition effective et non simplement potentielle ${ }^{71}$. En effet, la décision sur le régime belge d'exonération des bénéfices excédentaires indiquait que « le bénéfice exonéré ne pourrait jamais faire l'objet d'une double imposition $\gg{ }^{72}$. Eviter ainsi une double imposition potentielle - qui entraînerait une double non-imposition - serait contraire au principe de proportionnalité qui doit être observé pour justifier un avantage fiscal sélectif prima facie $e^{73}$.

En résumé, dans le cadre de ces deux procédures, la Commission semble examiner si la réduction ou l'exonération fiscale inconditionnelle et unilatérale dans un Etat membre, ce qui entraîne une situation de double non-imposition, résulte d'une législation fiscale ou d'une application de cette législation qui déroge au système commun d'imposition ${ }^{74}$. Si tel est le cas, une aide d'État est accordée (l'affaire belge); si tel n'est pas le cas, le droit des aides d'État ne permet pas de remédier à la situation de double non-imposition (McDonald's).

\section{Titre 2. Évaluation institutionnelle}

Les arguments avancés par la Commission dans les procédures décrites ci-dessus sont controversés et de large portée. Ils soulèvent de nombreuses questions, déjà débattues dès le début de ces procédures, mais qui n'ont pas reçu de réponse concluante à ce jour. Les critiques formulées à l'encontre des actions de la Commission sont généralement très techniques, dans la mesure où le sujet est abordé par une perspective strictement fondée sur l'interprétation correcte des conditions de l'article 107, paragraphe 1 TFUE et en particulier au regard de l'exigence de sélectivité ${ }^{75}$. Cependant, d'autres critiques plus fondamentales suggèrent que les actions de la Commission bouleversent tant les relations entre l'UE et les Etats Membres que les relations institutionnelles au sein de l'ordre juridique de l'UE. En effet, les parties qui ont intenté des recours contre les décisions de la Commission soulignent régulièrement une violation des articles 4 et 5 TUE et dénoncent une « harmonisation déguisée en matière fiscale ${ }^{76}$.

\footnotetext{
${ }^{71}$ Décision belge sur le traitement des bénéfices excédentaires, points 172-174. Voir aussi J. Rapp \& R. Ianus, op. cit., 155-156.

72 J. Rapp \& R. Ianus, op. cit., 155 (traduction libre).

${ }^{73}$ Décision belge sur le traitement des bénéfices excédentaires, point 175 . Voir aussi J. Rapp \& R. Ianus, op. cit., 156.

${ }^{74}$ Voy. également J. Rapp \& R. Ianus, op. cit., 148, qui soutiennent que la situation de double non-imposition ne devient pertinent que lorsque la Commission a déjà établi qu'un certain traitement déroge du système commun d'imposition (dans la même veine, J. Rapp \& R. Ianus, op. cit., 148).

${ }^{75}$ Voy. la doctrine abondante, par exemple, A. Giraud \& S. Petit, Tax Rulings and State Aid Qualification: Should Reality Matter?, European State Aid Law Quarterly 2017, 233-242; A. Gunn \& J. Luts, Tax rulings, APAs and state aid: legal issues, EC Tax Review 2015, 119-125; M. Lang, Tax rulings and state aid law, British Tax Review 2015, 391-395; L. Leclercq \& J. du Pasquier, Aides d'État à caractère fiscal: mieux comprendre pour mieux se defender: À propos des entreprises multinationals ayant bénéficié de rulings, Revue de Droit Fiscal 2015, 60-64; D.A. Kyriazis, From Soft Law to Soft Law Through Hard Law: The Commission's Approach to the State Aid Assessment of Tax Rulings, European State Aid Law Quarterly 2016, 428 et suiv.; R. Lyal, Transfer pricing rules and state aid, Fordham International Law Journal 2015, 1017-1043; S. Moreno González, State Aid and Tax Competition: Comments on the European Commission's Decisions on Transfer Pricing Rulings, European State Aid Law Quarterly 2016, 556 et suiv.; P. Nicolaides, State Aid Rules and Tax Rulings, European State Aid Law Quarterly 2016, 416 et suiv.; E. Raingeard De la Blétière, Les décisions individuelles à la lumière des aides d'Etat: coup de projecteur sur un risque méconnu, Revue de Droit Fiscal 2015, 51 et suiv.; P. Rosso-Maccanico, A New Framework for State Aid Review of Tax Rulings, European State Aid Law Quarterly 2015, 371 et suiv. et F. Jury, Le régime des aides d'État : un moteur efficace de la politique fiscale européenne, Rev. UE 2018, 427. Voy. également, pour plus d'orientations de la Commission elle-même, la communication de la Commission sur la notion d'aide d'Etat visée à l'article 107, paragraphe 1, du traité sur le fonctionnement de l'Union européenne, JO C 262 du 1 er, point 169 et suiv. et Working Paper on State Aid and Tax Rulings, http://ec.europa.eu.

${ }^{76}$ Voir notamment le troisième moyen soulevé dans l'affaire Luxembourg c. Commission, T-516/18; le troisième moyen soulevé dans l'affaire Luxembourg c. Commission, T-816/17 ; le huitième moyen soulevé dans l'affaire Irlande c. Commission, T-778/16 ; le premier moyen dans l'affaire SJM Centre de coordination c. Commission, T-420/16; le quatorzième moyen dans l'affaire Apple Sales International et Apple Operations Europe c.
} 
L'objet de cet article est d'analyser le bien-fondé de ce dernier type de critique et, partant, d'évaluer les actions de la Commission d'un point de vue institutionnel. L'analyse institutionnelle utilisée dans la présente contribution se concentre premièrement sur des questions liées à la structure verticale (i.e. la relation constitutionnelle entre les Etats membres et l'UE) et en particulier aux effets du droit des aides d'Etat sur la compétence des Etats membres en matière de la fiscalité directe (cf. Chapitre 1). Deuxièmement, elle s'oriente vers la structure horizontale (i.e. les relations entre les institutions de l'ordre juridique de l'UE ${ }^{77}$ ) qui examinera en particulier la relation constitutionnelle entre les pouvoirs de l'UE en vertu du droit des aides d'Etat et le rapprochement des législations (cf. Chapitre 2).

\section{Chapitre 1. Structure verticale}

L'aspect vertical de l'analyse concerne les effets du droit des aides d'Etat sur la compétence des Etats membres en matière de la fiscalité directe (supra). À notre avis, cette question peut être abordée dans une perspective ascendante et descendante. La perspective ascendante part de la constatation que la fiscalité directe relève, en principe, de la compétence des États membres (supra) et examine si cette constatation implique qu'un Etat membre peut invoquer sa souveraineté en matière de fiscalité directe pour s'opposer à une application du droit des aides d'Etat (cf. Section 1). L'approche descendante, en revanche, part des dispositions du droit communautaire des aides d'État et examine les limites des effets que de telles dispositions peuvent avoir sur la compétence fiscale des États membres (cf. Section 2).

\section{Section 1. Perspective ascendante}

Comme décrit ci-dessus, en l'état actuel du droit de l'UE, le domaine de la fiscalité directe semble relever de la catégorie des « compétences non attribuées à l'Union dans les traités » ou des « compétences retenues par les Etats membres». Dans cette perspective, plusieurs Etats membres visés dans les récentes procédures relatives à des rescrits fiscaux ont émis des critiques, faisant valoir en substance que la Commission empiète indûment sur leur souveraineté en matière de la fiscalité directe ${ }^{78}$.

Les mérites d'un tel argument sont, à première vue, discutables. En effet, comme mentionné cidessus, les « compétences retenues par les Etats membres », dont la fiscalité directe fait partie, doivent s'exercer conformément au droit de l'UE y compris le droit des aides d'Etat ${ }^{79}$. La CJUE a fondé cette position tant sur "l'effet utile » du droit de l'Union que sur le principe de

Commission, T-892/16; le huitième moyen dans l'affaire Irlande c. Commission, T-778/16. L'analyse des arguments développés par les parties dans leurs pourvois est limitée aux documents publiés sur le site de la Cour de Justice à la date du 10 décembre 2018.

${ }^{77}$ Pour cette distinction, voir par exemple K. Lenaerts, Le juge et la Constitution aux Etats-Unis d'Amérique et dans l'ordre juridique européen, Bruxelles, Bruylant, 1988, 248, no. 237 et pareillement A. von Bogdandy \& J. Bast, The Federal Order of Competences, in A. von Bogdandy \& J. Bast (dir.), Principles of European Constitutional Law, Munich, Beck, 2009, 284.

${ }^{78}$ Voy. par exemple, le troisième moyen invoqué par le Luxembourg dans sa demande d'annulation de la décision Amazon (T-816/17) (« la Commission procèderait en réalité à une instrumentalisation du droit des aides d'État [...], en violation de la compétence exclusive des États membres en matière de fiscalité directe ») et du huitième moyen de l'Irlande dans sa demande d'annulation de la décision Apple (T-778/16) (« [le] défaut de compétence de la Commission s'agissant d'adopter la décision attaquée et de la violation par la Commission des articles 4 et 5 TUE et du principe de l'autonomie fiscale des États membres »). Des arguments similaires ont déjà été avancés dans la doctrine, voy. par exemple T. Lübbig, L'application de l'article $87 \mathrm{du}$ Traité de Rome aux aides fiscales: un coup d'Etat communautaire?, RMCUE 2003, 124 et suiv.

${ }^{79}$ CJUE 29 mars 2012, 3M Italia, C-417/10, publié(e) au Recueil numérique (Recueil général), point 25 et CJCE 15 juil. 2004, Espagne c/ Commission, C-501/00, Rec. I-06717, point 123. Voir aussi TPICE 27 janv. 1998, Ladbroke Racing c/ Commission, T-67/94, Rec. II-00001, point 54 et Trib. UE 25 mars 2015, Belgique c/ Commission, T-538/11, publié(e) au Recueil numérique (Recueil général), point 66. 
coopération loyale (article 4, paragraphe 3 , du TUE) ${ }^{80}$. Par conséquent, bien que l'Union ne dispose d'aucune compétence dans la matière de la fiscalité directe (voir ci-dessus), « l'exercice effectif des compétences communautaires ne saurait être limité « de quelque façon » par l'existence d'une compétence nationale ${ }^{81}$. Ainsi, il est normal que « la Cour [tende] à assurer l'effectivité du droit de l'Union par « des incursions de la compétence communautaire dans les souverainetés nationales là où elles sont nécessaires », au sens de l'arrêt De Gezamenlijke Steenkolenmijnen ${ }^{82}$. Par conséquent, il semble qu'aucun argument constitutionnel ne peut empêcher l'application du droit des aides d'Etat dans le domaine de la fiscalité directe. Pour emprunter les mots du prof. Lenaerts, « il n'y a tout simplement pas de noyau de souveraineté que les États membres puissent invoquer, comme tel, contre l'[UE] $\gg^{83}$.

Néanmoins, l'argument selon lequel la Commission empiétait indûment sur les compétences des Etats membres en matière de la fiscalité directe pourrait encore bénéficier d'un certain mérite. En effet, la jurisprudence indiquant que les compétences retenues doivent être exercées conformément au droit de l'Union, souligne que le champ d'application du droit de l'Union est limité. Comme cela a été mis en évidence par la doctrine, cette jurisprudence implique - en fait - qu'il faut établir une distinction cruciale entre l'existence d'une compétence (en matière de la fiscalité directe) pour les Etats membres et l'exercice de cette compétence par les Etats membres, seul l'exercice est « encadré » par le droit de l'UE (des aides d'Etat) ${ }^{84}$. En d'autres termes, l'action de l'UE dans le domaine des aides d'Etat « ne devrait pas remettre en cause l'existence de cette compétence $»^{85}$. Par conséquent, en référence à la notion de souveraineté fiscale visée plus haut, cela impliquerait que les Etats membres conservent tant leur compétence en matière d'imposition objective que territoriale ${ }^{86}$.

\footnotetext{
${ }^{80}$ T. Ronse, Les compétences de l'Union européenne, Commentaire J. Mégret, Bruxelles, Editions de l'Université de Bruxelles, 2017, 145, no. 154.

${ }^{81}$ T. Ronse, op. cit., 149 , no. 158 .

${ }^{82}$ T. Ronse, op. cit., 150, no. 160, en référence à CJCE 23 févr. 1961, De Gezamenlijke Steenkolenmijnen in Limburg c/ Haute Autorité, 30/59, Rec. I-00003, point 24, dans lequel le système du contrôle des aides d'Etat était décrit comme «permettre des incursions de la compétence communautaire dans les souverainetés nationales là où elles sont nécessaires pour que, du fait des pouvoirs retenus par les États membres, l'effet utile du traité ne soit pas grandement diminué et sa finalité gravement compromise ». Voy. aussi Trib. UE 25 mars 2015, Belgique c/ Commission, T -538/11, publié(e) au Recueil numérique (Recueil général), point 65.

${ }^{83}$ K. Lenaerts, Constitutionalism and the Many Faces of Federalism, American Journal of Comparative Law 1990, no. 38, 220 (traduction par V. Constantinesco, La souveraineté est-elle soluble dans l'Union européenne?,
} L'Europe en Formation 2013, vol. 368, no. 2, 119-135).

${ }^{84}$ Pour la juxtaposition du droit européen et des « compétences retenues » en général, voir D. Simon, Le système juridique communautaire, Paris, PUF, 2001, 134, no. 91; L. Azoulai, op. cit., 343; V. Constantinesco et V. Michel, Compétences de l'Union européenne, Rép. de droit européen, no. 168; M. Blanquet, Droit général de l'Union européenne, Paris, Dalloz, 2018, 106, no. 153 et T. Ronse, op. cit., 150, no. 160. Pour la juxtaposition de la législation de l'UE en général, mais spécifiquement des dispositions relatives à la libre circulation et à la fiscalité directe, voir K. Lenaerts \& L. Bernardeau, L'encadrement communautaire de la fiscalité directe, CDE 2007, 30; D. Berlin, Politique fiscale (volume II), Commentaire J. Mégret, Bruxelles, Editions de l'Université de Bruxelles, 2012, 25, no. 1057 et D. Weber, op. cit., 586. Pour la juxtaposition du droit des aides d'Etat et de la fiscalité directe, voir K. Lenaerts, L'encadrement par le droit de l'Union européenne des compétences des membres, in Mélanges en l'honneur de Jean-Paul Jacqué, Paris, Dalloz, 2010, 438-442 et en particulier nos. 56-57; I. Papadamaki, Les aides d'État de nature fiscale en droit de l'Union européenne, Bruxelles, Bruylant, 2018, 449 et suiv. et A. Maitrot de la Motte, Droit fiscal de l'Union européenne, Bruxelles, Bruylant, 2016, 286. Pour une critique de cette distinction, voy. B. De Witte, Exclusive Member State Competences, in S. Garben \& I. Govaere (dir.), The Division of Competences between the EU and the Member States, Londres, Bloomsbury Publishing, 2017, 62 et J.E. Sullivan, The Non-Exercise of Taxing Powers by Member States and Its Compatibility with EC Law, European Taxation 2009, 189 et suiv.

${ }^{85}$ I. Papadamaki, op. cit., 452, no. 858. Voyez aussi T. Ronse, op. cit., 150, no. 160.

${ }^{86} \mathrm{Il}$ semble que cette conclusion est partagée par la Commission (Communication de la Commission relative à la notion d' « aide d'État» visée à l'article 107, paragraphe 1, du traité sur le fonctionnement de l'Union européenne, JOUE 2016 C 262, p. 1, point 156: «Les États membres sont libres de décider de la politique économique qu'ils 
Sur la base de ces considérations, nous tirons des conclusions prudentes en ce qui concerne certains arguments de la Commission.

- Premièrement, en ce qui concerne les cas de l'évasion fiscale, la Commission semble parfois empiéter sur le pouvoir d'imposition objectif des Etats membres. Cela concerne, primo, son raisonnement selon lequel les Etats membres ont octroyé des aides en n'adoptant pas, dans le cadre de leurs systèmes fiscaux nationaux, des règles anti-abus de base(par exemple, des règles concernant les prix de transfert). Après tout, ne pourraiton soutenir que le fait de ne pas adopter de règles anti-abus constitue par excellence la forme d'une absence d'exercice de la compétence en matière de fiscalité directe ${ }^{87}$ ? Cet argument s'applique également aux cas où les Etats membres n'ont pas adopté, du point de vue de la Commission, des règles anti-abus suffisamment efficaces ; après tout, n'est-ce pas là un cas d'une définition du pouvoir d'imposition objectif ${ }^{88}$, qui échappe au contrôle de l'UE en matière d'aides d'Etat ${ }^{89}$ ?

- Deuxièmement, les arguments que la Commission utilise dans le cadre d'un arbitrage fiscal semblent parfois porter atteinte au pouvoir d'imposition territorial des Etats membres. En effet, si les Etats membres restent capables de définir les limites territoriales de leurs systèmes de fiscalité directe du point de vue de l'UE, ils ne peuvent être tenus responsables des problèmes liés aux avantages fiscaux sélectifs découlant d'inadéquations ou de disparités car ils trouvent leur origine ou leur cause en dehors de leur système d'imposition directe ou en interagissant avec un autre système d'imposition directe ${ }^{90}$. Cette position semble être acceptée dans l'affaire McDonald's (voyez supra).

On peut toutefois se demander si la conclusion selon laquelle les États membres ont conservé la compétence en matière de fiscalité directe et seulement l'exercice de cette compétence est « encadré » par le droit primaire, implique effectivement « la reconnaissance au profit des Etats membres de l'existence des matières complètement imperméables à l'emprise du droit communautaire $»{ }^{91}$. Premièrement, la division entre l'existence et l'exercice d'une compétence est difficile à mettre en pratique, en ce sens qu'il est difficile de déterminer quand un Etat exerce ou définit simplement son pouvoir d'imposition ${ }^{92}$. Deuxièmement et plus fondamentalement, il

jugent la plus appropriée et, en particulier, de répartir comme ils l'entendent la charge fiscale entre les différents facteurs de production. Toutefois, les États membres doivent exercer cette compétence dans le respect du droit de l'Union ») et par la Cour (CJUE 26 avr. 2018, ANGED, C-233/16, publié(e) au Recueil numérique (Recueil général), point 51, dans lequel il est fait explicitement référence à la déclaration de la Commission).

${ }^{87}$ Le professeur Vanistendael fait également valoir que le pouvoir d'introduire des règles anti-abus est un élément essentiel de l'existence du pouvoir d'imposition objectif des Etats membres (F . Vanistendael, op. cit., 202).

${ }^{88}$ Les règles anti-abus d'un Etat membre reflètent l'équilibre entre deux principes juridiques contradictoires, à savoir la préservation de la sécurité juridique et la promotion de l'égalité (voy. H. Filipczyk, Tax Avoidance and Rationality of Law,Varsovie, Wolters Kluwer Polska, 2017, 193 et A. Saydé, op. cit., 167 et suiv.); un exercice qui constitue une décision souveraine de chaque État membre. Certains États membres attachent plus d'importance à la sécurité juridique et optent donc pour une approche dite du «form over substance ». D'autres États membres attachent plus d'importance au principe de l'égalité, imposant ainsi des impôts sur la base de l'opération économique sous-jacente, quel que soit le «manteau juridique» dans lequel ces transactions ont été intégrées («substance over form ») (voy. aussi F. Zimmer, General report, Form and Substance in Tax Law, Cah.Dr.F.Int., Volume LXXXVIIa, IFA 2002 Congrès d'Oslo, La Haye, Kluwer, 2002, 37).

${ }^{89}$ Cependant, une conclusion différente peut probablement s'imposer dans les cas où un État membre dévie d'une mesure nationale anti-abus existante, soit à titre d'exception législative, soit par suite d'une application inadéquate de cette disposition dans un cas particulier.

${ }^{90}$ Voyez en ce sens D. Weber, op. cit., 588 et 615 ; D. Berlin, op. cit., 25-26, nos. 1058-1059.

${ }^{91} \mathrm{~K}$. Boskovits, Le juge communautaire et l'articulation des compétences normatives entre la Communauté européenne et ses Etats membres, Bruxelles, Bruylant, 1999, 120.

${ }^{92}$ D. Berlin, op. cit., 26, no. 1059 et D. Weber, op. cit., 586. Ce qui a même amené certains auteurs à soutenir qu'un « non-exercice » des pouvoirs d'imposition directe devrait également relever du champ d'application du droit 
semble que lorsque le comportement d'un Etat membre dans le domaine de l'imposition directe tombe dans le champ d'application (matériel) du droit de l'UE (des aides d'Etat) ${ }^{93}$, il est considéré comme un exercice de pouvoir d'imposition ${ }^{94}$ et il est donc affecté par ce droit. Il semble donc que la compétence fiscale directe devrait plutôt être qualifiée de «compétence résiduaire » au lieu de " compétence réservée » ${ }^{95}$, ce qui n'existe « qu'après avoir examiné les dispositions des traités communautaires qui auraient pu faire échapper la matière en cause à la compétence des Etats membres $\gg{ }^{96}$.

\section{Section 2. Perspective descendante}

En abordant la question verticale d'une perspective descendante, la question est de savoir si les effets du droit des aides d'État sur la compétence fiscale des États membres sont constitutionnellement limités. Cette question peut également être abordée sous deux angles, selon que l'on considère le droit des aides d'État comme conférant une compétence à l'Union (§1.) ou comme de simples obligations de fond incombant aux États membres $(\S 2 .)^{97}$.

\section{§1. Approche en termes de compétence}

Certains auteurs semblent aborder les dispositions du droit des aides d'État sous l'angle des compétences accordées à $1^{1} \mathrm{UE}^{98}$. Suite à un tel raisonnement, il ne fait aucun doute que, en vertu du principe d'attribution, une compétence dans le domaine des aides d'Etat a été attribuée à l'UE par les articles 107 à 109, du TFUE ${ }^{99}$ pour atteindre l'objectif de l'article 3, paragraphe 3, du TUE ${ }^{100}$. En particulier, l'article 108, paragraphe 2, du TFUE attribue à la Commission une « compétence de contrôle » qui lui permet de se prononcer sur la compatibilité des aides d'État par voie de décision individuelle ${ }^{101}$.

de l'Union (des aides d'Etat) (J.E. Sullivan, The Non-Exercise of Taxing Powers by Member States and Its Compatibility with EC Law, European Taxation 2009, 189 et suiv.).

${ }^{93}$ Une analyse de fond sort du cadre de cette contribution.

${ }^{94}$ En ce sens, K. Lenaerts, L'encadrement par le droit de l'Union européenne des compétences des membres, in Mélanges en l'honneur de Jean-Paul Jacqué, Paris, Dalloz, 2010, 442.

${ }^{95} \mathrm{~K}$. Lenaerts, Le juge et la Constitution aux Etats-Unis d'Amérique et dans l'ordre juridique européen, Bruxelles, Bruylant, 1988, 461, no. 389 (et aussi 481 et seq., nos. 406 et seq.) ; P. Van Nuffel, De rechtsbescherming van nationale overheden in het Europees recht, Kluwer, Deventer, 2000, 111.

${ }^{96} \mathrm{~K}$. Lenaerts, Le juge et la Constitution aux Etats-Unis d'Amérique et dans l'ordre juridique européen, Bruxelles, Bruylant, 1988, 461, no. 389.

${ }^{97}$ Certains estiment en effet que les dispositions du droit des aides d'État sont des dispositions conférant une compétence à l'Union européenne (voir infra), tandis que d'autres considèrent ces dispositions comme de simples interdictions (voir infra). Les auteurs n'entendent pas résoudre ce débat dans le cadre de la présente contribution et ont décidé d'aborder la question sous les deux angles.

${ }^{98}$ Par exemple, M. Peeters, Lidstatelijke steunmaatregelen in de Europese Unie, Bruges, Die Keure, 2012, 155 et, si nous comprenons bien les auteurs, H.P. Ipsen, Europaïsches Gemeinschaftsrecht, Tübingen, Mohr Siebeck, 1972, 415, no. 3; J. Winter, Nationale steunmaatregelen en het Gemeenschapsrecht, Kluwer, Deventer, 1981, 116, no. 96 ; T. Joris, Nationale steunmaatregelen en het Europees Gemeenschapsrecht, Antwerpen, Maklu, 1994,230 et seq. et J.-P. Keppenne, Guide des aides d'état en droit communautaire, Bruxelles, Bruylant, 1999, 9, no. 7. Voir également les auteurs qui considèrent que les dispositions du droit des aides d'État confèrent une « compétence de contrôle » à l'UE (par exemple, M. Blanquet, op. cit., 74, no. 93 et pareillement R. Schütze, European Union Law, Oxford, Oxford University Press, 2012, 327 et seq.).

${ }^{99}$ Voir infra pour une discussion sur la nature de cette compétence.

100 «L'Union établit un marché intérieur». Voir aussi le Protocole no. 27 : «[L]e marché intérieur tel qu'il est défini à l'article 3 du traité sur l'Union européenne comprend un système garantissant que la concurrence n'est pas faussée ».

${ }^{101}$ M. Blanquet, op. cit., 74, no. 93 et O. Peiffert, L'application du droit des aides d'État aux mesures de protection de l'environnement, Bruxelles, Bruylant, 2015, 275, no. 490. 
La question est toutefois de savoir s'il y a des limites à l'exercice de pouvoirs au sein de cette compétence ${ }^{102}$ ou, autrement dit, si le droit des aides d'Etat ne devrait pas s'étendre à certains domaines de compétence dans le domaine de la fiscalité directe nationale. En effet, les parties qui ont intenté des recours contre les décisions de la Commission soulignent régulièrement une violation des articles 4 et 5 TUE (voir supra), qui incluent notamment les principes de subsidiarité (a.), de proportionnalité et de coopération loyale (b.). Nous proposons d'examiner si ces principes sont effectivement susceptibles d'affecter l'intensité du contrôle exercé par la Commission sur les actions des États membres dans le domaine de la fiscalité directe.

\section{a. Subsidiarité}

Le principe de subsidiarité, consacré à l'article 5, paragraphe 3, du TUE, ne concerne pas la question de savoir si l'UE $a$ une compétence ou non, mais si l'UE peut ou doit exercer cette compétence ${ }^{103}$. En effet, en vertu du principe de subsidiarité, même si l'UE disposait d'une compétence in abstracto dans un domaine (comme les aides d'État), elle ne pourrait agir in concreto que si les objectifs visés par l'action ne peuvent pas être atteints de manière suffisante par les États membres (test absolu et négatif) et pourraient être mieux réalisés au niveau de l'UE (test relatif et positif) ${ }^{104}$. On peut se demander si ce principe de subsidiarité peut empêcher la Commission d'exercer ses prérogatives dans le cadre du contrôle des aides d'Etat.

À cet égard, la plupart des auteurs défendent la position prima facie logique selon laquelle il faut répondre à cette question par la négative. Après tout, le droit de la concurrence, qui inclut le droit des aides d'Etat, est considéré comme relevant des compétences exclusives de l'UE (article 3, paragraphe 1, point b), du TFUE). Par conséquent, comme l'article 5, paragraphe 3, du TUE prévoit que le principe de subsidiarité s'applique uniquement dans les domaines qui ne relèvent pas de la compétence exclusive de l'UE, les actions de la Commission dans le domaine des aides d'Etat ne peuvent jamais être restreintes en fonction du principe de subsidiarité ${ }^{105}$. Cependant, l'étendue de la compétence exclusive de l'UE dans le domaine du droit de la concurrence, et donc le champ d'application du principe de subsidiarité, sont contestés. Selon certains auteurs, qui trouvent un appui dans le libellé de l'article 3, paragraphe 1 , point $\mathrm{b}$ ), du TFUE, qui se réfère uniquement à «l'établissement des règles de concurrence nécessaires au fonctionnement du marché intérieur », la compétence exclusive ne porte que sur la compétence de légiférer, à l'exclusion de la compétence d'administrer les règles ${ }^{106}$. Sur la

${ }^{102}$ Cela remonte à la distinction traditionnelle établie entre les questions qui concernent (i) la délimitation de la compétence de l'UE et (ii) l'exercice de pouvoirs au sein de cette compétence. Voir article 5, paragraphe 1, du TUE et, par exemple, M. Blanquet, op.cit., 73, no. 91 et C. Blumann \& L. Dubouis, Droit institutionnel de l'Union européenne, Paris, LexisNexis, 2016, 442 et 495.

${ }^{103}$ Par exemple, Kapteyn et al., Kapteyn \& VerLoren van Themaat. The Law of the European Union and the European Communities, Alphen aan den Rijn, Kluwer Law International, 2008, 141; A. Dashwood et al., Wyatt and Dashwood's European Union Law, Oxford, Hart Publishing, 2011, 115; M. Blanquet, op. cit., 114, no. 162; T. Ronse, op. cit., 179, no. 196 et M. Dony, Droit de l'Union européenne, Bruxelles, Editions de l'Université de Bruxelles, 2018, 91, no. 146, selon lequel le principe « a pour objet de dissocier « compétence » et «pouvoirs »: même si l'Union est compétente dans une matière, elle ne peut pas automatiquement faire usage de tous les pouvoirs nécessaires pour exercer cette compétence ».

104 R. Schütze, European Union Law, Cambridge, Cambridge University Press, 2018, 257 et C. Blumann \& L. Dubouis, op. cit., 499-500.

${ }^{105}$ M. Peeters, op. cit., 157, no. 308; M. Dony, op. cit., 91, no. 146.

${ }^{106}$ C. Calliess, Art. 3 AEUV, in C. Calliess \& M. Ruffert (dir.), EUV/AEUV, Munich, Beck, 2016, no. 9; M. Nettesheim, Art. 3 AEUV, in E. Grabitz, M. Hilf \& M. Nettesheim, Das Recht der Europäischen Union: EUV/AEUV, Munich, Beck, 2018, no. 14; W. Obwexer, Art. 3 AEUV, in H. von der Groeben, J. Schwarze \& A. Hatje, Europäisches Unionsrecht, Munich, Beck, 2015, no. 14; M. Monti, Legislative and Executive Competences in Competition Law, in L. Azoulai (dir.), The question of competence in the European Union, Oxford, Oxford University Press, 2014, 102 et C. Blumann \& L. Dubouis, op. cit., 459. On peut noter que même M. Dony, qui soutient que le principe de subsidiarité ne peut jamais être appliqué dans le droit des aides d'État (voyez note 106), 
base de ce qui précède, certains auteurs ont conclu que l'administration du droit de la concurrence (incl. l'article 107, paragraphe 1, du TFUE ${ }^{107}$ ) par la Commission est, en principe, soumise au principe de « subsidiarité exécutive ${ }^{108}$.

Sur cette base, certains auteurs ont estimé que la Commission a violé le principe de subsidiarité en concluant que les autorités fiscales de l'Etat membre concerné n'ont pas correctement appliqué la législation nationale (anti-abus) dans des cas individuels, ce qui a entraîné un avantage sélectif pour les entreprises concernées. En effet, certains auteurs nient le fait que la Commission est la mieux placée ${ }^{109}$ pour se charger de la police de l'application des dispositions fiscales nationales dans des cas individuels, comme elle semble le faire dans les récentes procédures en matière fiscale. En effet, les autorités nationales compétentes disposent en général de connaissances spécialisées relatives à l'application de la législation fiscale nationale. Quigley, par exemple, soutient «qu'il ne serait pas souhaitable que la Commission cherche à vérifier si les autorités fiscales nationales appliquent toujours correctement les dispositions fiscales pertinentes [parce que cela serait contraire] au principe de subsidiarité $\gg{ }^{110}$. Il ajoute, en ce qui concerne les récentes procédures sur les rescrits fiscaux, qu'il « est douteux que la Commission soit la mieux placée pour tenter de deviner la position des autorités fiscales nationales dans leur évaluation des accords de prix de transfert négociés avec des groupes multinationaux » et, par conséquent, même dans le cas où un avantage économique serait conféré, « si les règles relatives aux aides d'Etat devraient même s'appliquer ${ }^{111}$.

Nous estimons néanmoins pour les raisons suivantes que le principe de subsidiarité ne doit pas empêcher la Commission d'examiner si l'application de la législation fiscale par les autorités nationales dans un cas particulier revient à octroyer des aides d'Etat. Premièrement, accepter la position selon laquelle le droit des aides d'Etat ne devrait pas s'appliquer dans le contexte, par exemple, de l'application de la législation fiscale en matière de prix de transfert (supra) ouvrirait la porte à des abus. Cela créerait des domaines de droit, qui seraient de facto exclus du champ d'examen des aides d'Etat, et en limiterait ainsi l'effet utile. Deuxièmement, en lien avec cette idée, le contrôle de l'application de la législation fiscale ne peut réellement pas être « réalisé de manière suffisante par les Etats membres [et ne peut pas] être mieux réalisé au niveau de l'Union » (article 5, paragraphe 3, du TUE). En fait, en supposant que les autorités nationales n'ont volontairement pas appliqué correctement la législation fiscale nationale, conférant ainsi un avantage économique à une entreprise, la correction ne serait probablement pas mieux

admet que l'administration du droit de la concurrence n'est pas couverte par l'exclusivité (M. Dony, op. cit., 81, no. 127).

${ }^{107}$ Néanmoins, de nombreux auteurs - même ceux qui admettent que l'administration du droit de la concurrence 7 n'est pas couverte par l'exclusivité (voyez note 107) - soutiennent qu'une partie de la compétence (de la Commission) pour administrer le système de droit des aides d'Etat est exclusive, notamment la compétence pour contrôler l'application des dérogations au principe d'incompatibilité des aides, telle qu'elle figure principalement à l'article 107, paragraphes 2 et 3, du TFUE (O. Peiffert, op. cit., 275, no. 490 juncto no. 283 et C. Blumann \& L. Dubouis, op. cit., 464). La Cour semble partager cette opinion (Trib. UE 26 nov. 2015, Espagne c/ Commission, T-461/13, publié(e) au Recueil numérique (Recueil général), point 182, en référence à CJUE 19 déc. 2012 , Mitteldeutsche Flughafen et Flughafen Leipzig-Halle c/ Commission, C-288/11, publié(e) au Recueil numérique (Recueil général), point 79).

${ }^{108}$ Comparez avec R. Schütze, European Union Law, Cambridge, Cambridge University Press, $2018,341$.

${ }^{109}$ Le test relatif absorbe généralement le test absolu (C. Blumann \& L. Dubouis, op. cit., 501).

${ }^{110}$ C. Quigley, European State Aid Law and Policy, Oxford, Hart Publishing, 2015, 10 et 106. Voyez pour une position comparable, W. Schön, Taxation, in L. Hancher, T. Ottervanger \& P.J. Slot, EU State Aids, Londres, Sweet \& Maxwell, 2016, 411, no. 13-040.

${ }^{111}$ C. Quigley, Differential Tax Treatment, Tax Rulings and EU State Aid Law, in R. Danon (dir.), Base Erosion and Profit Shifting. Impact for European and international tax policy, Genève, Schulthess, 2016, 472. Cette position semble s'appuyer sur la jurisprudence britannique (R v. Attorney General, ex parte ICI [1987], 1 Common Market Law Reports 72). 
réalisée au niveau national, mais plutôt à un niveau supranational ${ }^{112}$. Troisièmement, il semble que la portée du principe de subsidiarité exécutive est limitée. Dans une affaire de droit de la concurrence, le Tribunal de l'Union a en effet confirmé que, par essence, ce principe ne saurait priver la Commission d'une compétence pour appliquer directement les dispositions des traités de l'Union dans des cas individuels ${ }^{113}$.

\section{b. Proportionnalité et coopération loyale}

Même si le principe de subsidiarité (exécutive) ne peut empêcher la Commission d'exercer ses compétences en matière de contrôle des aides d'Etat, on pourrait se demander (i) si le principe de proportionnalité, qui est incorporé à l'article 5, paragraphe 4, du TUE, constitue un frein à l'action en matière des aides d'Etat, ou (ii) si un tel frein pourrait résulter du principe de coopération loyale de l'article 4, paragraphe 3, du TUE.

Le principe de proportionnalité - qui porte aussi sur les compétences exclusives ${ }^{114}$ et administratives ${ }^{115}$, rendant inutile par conséquence la discussion ci-dessus - implique que « toute action de [l'UE] ne va pas au-delà de ce qui est nécessaire pour atteindre les objectifs [recherchée par l'action] ${ }^{116}$. À la différence du principe de subsidiarité, le principe de proportionnalité ne concerne pas la question de savoir si une compétence peut ou doit être exercée in concreto, mais bien (i) le degré d'intervention de l'exercice dans la sphère nationale et (ii) les moyens utilisés ${ }^{117}$. Du point de vue vertical, le premier élément pourrait être pertinent ${ }^{118}$. Bien qu'il faille reconnaître que le principe de proportionnalité ne peut imposer des limites fortes à l'action de la Commission dans le domaine des aides d'État, compte tenu des compétences (quasi) exclusives de cette dernière à cet égard (voir ci-dessus), certains auteurs semblent néanmoins soutenir qu'un certain seuil doit être dépassé pour conclure que, dans un cas particulier, l'(in)action d'un Etat membre équivaut à une aide d'Etat et qu'une intervention de la Commission dans la sphère du compétence de l'Etat membre ait justifiée ${ }^{119}$.

\footnotetext{
112 Cela concerne l'essence du contrôle des aides d'Etat par un organisme supranational, comme en témoigne l'article 67 du traité CECA de 1951. Voyez également Commission, XXIIe Rapport sur la politique de concurrence 1992, Luxembourg, Office des publications officielles des Communautés européennes, 1993, 84, no. $121:$ : Le contrôle des aides d'État est un domaine dans lequel le principe de subsidiarité a, par définition, peu de place. Il est en effet difficile d'imaginer qu'un État membre puisse contrôler, au nom de l'intérêt communautaire, les aides qu'il accorde lui-même sur son propre territoire ». Pour aller encore plus loin, on pourrait affirmer que la relation entre le contrôle des aides d'État par la Commission ou par les États membres est de nature complémentaire et non alternative, de sorte que le principe de subsidiarité ne s'applique pas. (Voir, par exemple, C.W.A. Timmermans, Judicial Protection against the Member States: Articles 169 and 177 Revisited, in D. Curtin \& T. Heukels, Institutional Dynamics of European Integration. Essays in honour of Henry G. Schermers. Volume II, Dordrecht, Kluwer, 1994, 392-393 et 405).

113 TPICE 8 mars 2007, T-339/04, France Télécom c/ Commission, Rec. II-00521, points 73 et 89.

${ }^{114}$ Par exemple, Kapteyn et al., op. cit., 145 ; M. Blanquet, op. cit., 123, no. 172 et C. Blumann \& L. Dubouis, op. cit., 511.

${ }^{115}$ C. Blumann \& L. Dubouis, op. cit., 512.

${ }^{116}$ K. Lenaerts \& P. van Ypersele, Le principe de subsidiarité et son contexte : étude de l'article 3 B du Traité CE, CDE 1994, 3 point 78, cité par Kapteyn et al., op. cit., 145 (traduction libre).

${ }^{117}$ Par exemple, Kapteyn et al., op. cit., 145 et A. Dashwood et al., 123. Voyez aussi C. Blumann \& L. Dubouis, op. cit., 512 et M. Blanquet, op. cit., 124-125, no. 172-173.

${ }^{118}$ Le deuxième élément (les moyens utilisés) concerne principalement la perspective horizontale (voyez infra). Comme souligné par le professeur Lenaerts, "cette répartition (horizontale) des compétences influence les relations verticales entre l'Union et les États membres »; en effet, « dans les cas où la procédure requiert la décision unanime en Conseil, chaque État membre dispose d'un droit de veto » (K. Lenaerts \& P. Van Nuffel, European Union Law, Sweet \& Maxwell, London, 2011, 115).

${ }^{119}$ M. Peeters, op. cit., 157-158, no. 309, qui soutient que la Commission ne peut exercer ses compétences de telle manière que les rares ressources dont elle dispose pour exercer son pouvoir de contrôle sont déployées d'une manière manifestement inappropriée pour atteindre l'objectif de l'article 107, paragraphe 1, du TFUE.
} 
Le principe de coopération loyale est, selon la Cour, un principe général « imposant aux Etats membres et aux institutions communautaires des devoirs réciproques de coopération loyale ${ }^{120}$, qui est actuellement consacré à l'article 4 , paragraphe 3 , première alinéa, du TUE. Le principe de coopération loyale comprend certes le devoir de l'Union de respecter les intérêts élémentaires des Etats membres ${ }^{121}$. Dans cette perspective, de nombreux auteurs ont soutenu que le principe de coopération loyale pourrait également fonctionner comme un principe régulateur de l'exercice des compétences de $1^{\prime} \mathrm{UE}^{122}$. En outre, les institutions de l'Union doivent, dans l'exercice de leurs compétences, tenir compte des effets sur les compétences des États membres, ce qui peut donner lieu à des obligations d'omission ${ }^{123}$. Une interdiction pèse dans cette perspective - sur les interventions disproportionnées qui nient les compétences retenues par des Etats membres ${ }^{124}$. Le principe de coopération nécessite donc un équilibre approprié entre d'une part l'efficacité du droit de l'Union et, d'autre part, le respect des compétences fondamentales nationales ${ }^{125}$.

Sur la base des principes de proportionnalité et de coopération loyale, dans le cadre du contrôle des aides d'Etat, la question se pose concernant la capacité de la Commission, à substituer son propre point de vue sur la manière dont la loi devrait s'appliquer, sans tenir compte des appréciations des autorités nationales. La réponse semble dépendre de la clarté de la loi et de la « marge d'interprétation » qui en résulte pour les autorités fiscales nationales. En effet, comme le soutient le professeur Azizi, l'étendue et la profondeur du contrôle des aides d'Etat par la Commission par rapport aux mesures des Etats membres sont fonction du degré d'autonomie laissé aux Etats membres par le cadre juridique pertinent ${ }^{126}$. En effet, dans ces cas où « une grande marge de liberté est laissée à l'apport de la volonté autonome des États Membres, [par exemple, dans le cas de compétences des États membres ne relevant pas du champ d'application du traité,] la portée et l'étendue du contrôle exercé par la Commission pourraient être réduites à une erreur manifeste $»^{127}$.

À cet égard, il convient de rappeler que les Etats membres ont, en principe, conservé leurs compétences fiscales et qu'il leur reste un certain degré de liberté. En outre, l'interprétation et l'application de la législation fiscale (par exemple les prix de transfert ou des règles antiabus) sont le plus souvent (et certainement dans les cas de décisions fiscales récentes devant la Commission) peu claires ${ }^{128}$. On pourrait donc également dire à cet égard que les Etats membres

\footnotetext{
${ }^{120}$ Par exemple, CJCE 10 févr. 1983, Luxembourg c/ Parlement, 230/81, Rec. I-255, point 37 et CJCE 16 oct. 2003, Irlande c/ Commission, C-339/00, Rec. I-11757, point 71.

${ }^{121}$ C. Calliess et al., Art. 4 EUV, in C. Calliess \& M. Ruffert (dir.), EUV/AEUV, Munich, Beck, 2016, no. 110.

${ }^{122}$ Par exemple, M. Blanquet, Droit général de l'Union européenne, Paris, Dalloz, 2018, 128-129; C. Calliess et al., Art. 4 EUV, in C. Calliess \& M. Ruffert (dir.), EUV/AEUV, Munich, Beck, 2016, no. 111 ; W. Obwexer, Art. 4 EUV, in H. von der Groeben, J. Schwarze \& A. Hatje, Europäisches Unionsrecht, Munich, Beck, 2015, no. 142 et A. Hatje, Art. 4 EUV, in J. Schwarze, EU-Kommentar, Nomos, Baden-Baden, 2012, no. 76.

${ }^{123}$ W. Obwexer, Art. 4 EUV, in H. von der Groeben, J. Schwarze \& A. Hatje, Europäisches Unionsrecht, Munich, Beck, 2015, no. 142 et A. Hatje, Art. 4 EUV, in J. Schwarze, EU-Kommentar, Nomos, Baden-Baden, 2012 , no. 76, en référence à CJCE 28 nov. 1991, Luxembourg c/ Parlement, 213/88, Rec. I-05643, point 29.

${ }^{124}$ Voyez C. Calliess et al., Art. 4 EUV, in C. Calliess \& M. Ruffert (dir.), EUV/AEUV, Munich, Beck, 2016 , no. 111. Cela montre que le principe est lié au principe de proportionnalité.

125 Ibid.

126 J. Azizi, The Tension between Member States' Autonomy and Commission Control in State Aid Matters: Selected Aspects, in H. Kanninen, N. Korjus \& A. Rosas (dir.), EU Competition Law in Context: Essays in Honour of Virpi Tiili, Oxford, Hart Publishing, 2009, 313.

127 J. Azizi, op. cit., 313 (traduction libre).

${ }^{128}$ Par exemple, A. Gunn \& J. Luts, op. cit., 121 ; R. Luja, General report, in J.L. da Cruz Vilaça et al. (dir.), Taxation, State aid and distortions of competition, Conference proceedings XXVIII FIDE Congress, Coimbra, Almedina, 2018, 58; M. Lang, Margin.
} 
disposent d'une certaine marge d'interprétation ${ }^{129}$. À la lumière de ce qui précède, il serait peu souhaitable que la Commission intervienne et substitue son point de vue à celui des autorités nationales. Selon nous, la Commission ne devrait donc exercer qu'un contrôle limité et intervenir uniquement en cas de d'écart manifeste, c.-à-d. si l'administration a pris une position que qu'aucune administration raisonnable aurait pu adopter ${ }^{130}$. Bien qu'une confusion entre une indication quant la politique d'attribution des ressources et un indice du degré de contrôle envisagé, ce point de vue semble également avoir été accepté par la Commission dans son document de travail intitulé « Working Paper on State Aid and Tax Rulings », dans lequel elle reconnaît que « la DG Concurrence se concentre sur les cas de violation manifeste du principe de pleine concurrence $\gg{ }^{131}$.

\section{§2. Approche en termes d'obligation}

Selon certains auteurs, les effets du droit de l'UE sur les aides d'Etat ne devraient pas être examinés sous l'angle de la compétence de l'UE (voir supra); au contraire, les dispositions du droit des aides d'État doivent simplement être considérées comme une obligation (Rechtsmaßstab) qui s'impose aux Etats membres et à laquelle doivent être comparées les actions des États membres (dans le domaine de la fiscalité directe) $)^{132}$.

De ce point de vue, la question est de savoir si le droit des aides d'Etat est susceptible d'imposer des obligations positives (obligations de faire) aux Etats membres, ou s'il ne prévoit que des obligations négatives (obligations de ne pas faire). Ceci est important pour les récentes procédures de la Commission et les liens avec la question soulevée ci-dessus concernant le fait de savoir si un Etat membre a accordé des aides d'Etat par l'absence d'adoption ou d'application de règles adéquates anti-PFA. En effet, la Commission semble prétendre qu'une telle omission peut être constitutive d'une aide d'Etat et que le système de contrôle des aides d'Etat peut ainsi imposer de facto une obligation de faire aux Etats membres. La question est cependant de savoir si une telle conclusion est conforme au droit des aides d'Etat.

À cet égard, la plupart des auteurs font valoir que le droit des aides d'État ne crée que des obligations négatives (obligations de ne pas faire). Ces auteurs décrivent le système de contrôle des aides d'Etat comme impliquant une interdiction de mettre en œuvre une aide d'Etat qui n'a

\footnotetext{
${ }^{129}$ Ce qui est prima facie différent d'un « pouvoir discrétionnaire » (c'est-à-dire « un pouvoir [...] qui lui permet de déterminer les bénéficiaires ou les conditions de la mesure accordée »), qui donne ipso facto à présumer qu'un avantage sélectif est accordé en appliquant cette disposition (CJCE 29 juin 1999, DM Transport, C-256/97, Rec. I-03913, point 27 et TPICE 6 mars 2002, Diputación Foral de Álava c/ Commission, T-92/00 et T-103/00, Rec. II-01385, point 35). Pour une discussion sur la qualification d'une marge d'interprétation en tant que pouvoir discrétionnaire, voir M. Lang, Discretionary Power of Tax Authorities as a State Aid Problem, in W. Haslehner et al., EU Tax Law and Policy in the $21^{\text {st }}$ Century, Alphen aan den Rijn, Kluwer Law International, 2018,91 et seq. ${ }^{130}$ A. Gunn \& J. Luts, op. cit., 121 et F.P. Sutter, Das EG-Beihilfenverbot und sein Durchführungsverbot in Steuersachen, Wien, Linde, 2005, 67 et seq. Voyez également R. Luja, op. cit., 84.

${ }^{131}$ Working Paper on State Aid and Tax Rulings, http://ec.europa.eu, point 23 in fine.

132 O. Peiffert, op. cit., 283, no. 504, qui cite V. Constantinesco, Compétences et pouvoirs dans les Communautés européennes - Contribution à l'étude de la nature juridique des Communautés, LGDJ, 1974, 105. Voy. aussi H. Kube, Nationales Steuerrecht und europäisches Beihilfenrecht, in U. Becker \& W. Schön, Steuer- und Sozialstaat im europä̈schen Systemwettbewerb, Tübingen, Mohr Siebeck, 2005, 107, qui fait la distinction entre « Kompetenzen » et « Rechtsmaßstaben » et soutient que l'article 107 TFUE appartient à cette dernière catégorie. Cette position est également soutenue, si nous comprenons les auteurs correctement, par Prechal, De Vries et Van Eijken, qui établissent une distinction entre « les compétences de l'UE » et « le champ d'application du droit de l'UE » et considèrent que, indépendamment du fait qu'une compétence ait été conféré à l'UE, l'action des Etats membres peut entrer dans le champ d'application du droit de l'Union et, en particulier, les diverses interdictions du droit de l'UE (S. Prechal, S. De Vries and H. Van Eijken, The Principle of Attributed Powers and the Scope of EU Law, in L. Besselink, F. Pennings and S. Prechal (dir.), The Eclipse of Legality, Alphen aan den Rijn, Kluwer Law International, 2011, 215).
} 
pas été autorisée par la Commission ${ }^{133}$. Ainsi, tout comme les dispositions sur la libre circulation, le système de contrôle des aides d'Etat est généralement considéré comme une forme d' « intégration négative » qui ne prévoit que des obligations négatives vis-à-vis des Etats membres et ne peut donner lieu à des obligations positives ${ }^{134}$. En d'autres termes, le système de contrôle des aides d'Etat ne peut contraindre les Etats membres à agir; c'est une conclusion qui est conforme à la position selon laquelle un non-exercice des pouvoirs d'imposition ne relève pas de la compétence (européenne) de contrôle des aides d'Etat (voir ci-dessus) ${ }^{135}$. De ce point de vue, l'argument de plusieurs demandeurs devant la CJUE selon lequel la Commission opère « une harmonisation fiscale déguisée » peut sembler avoir un certain mérite.

Néanmoins, il faudra peut-être nuancer cette conclusion. En effet, certains auteurs ${ }^{136}$, et même la Commission ${ }^{137}$, ont estimé que les aides d'État peuvent être accordées par une omission d'un État membre ${ }^{138}$. Cette position est prima facie conforme à l'approche adoptée en ce qui concerne les dispositions relatives à la libre circulation, dans le contexte de laquelle la CJUE a soutenue qu'une omission de la part d'un Etat membre peut produire une infraction d'une disposition formulée comme une interdiction, en liaison avec le principe de coopération loyale (ainsi créant une forme d' "obligation positive ») ${ }^{139}$. On pourrait donc se demander si le système de contrôle des aides d'Etat comporte à son tour, non seulement une obligation négative (c.à.d. une interdiction d'octroyer une aide), mais aussi une obligation positive (c.à.d. une obligation de prendre des mesures garantissant qu'aucune aide ne soit accordée) $)^{140}$.

${ }^{133}$ De nombreux auteurs soutiennent (explicitement ou implicitement) que la sanction de l'« incompatibilité » des
aides d'État ex article 107, paragraphe 1, du TFUE se réduit de facto à une interdiction (par exemple, K. Bacon,
European Union Law of State Aid, Oxford, Oxford University Press, 2017, 11-12, no. 1.22; A. Bartosch, EU-
Beihilfenrecht, Munich, Beck, 2016, 2; A. Evans, European Community Law of State Aid, Oxford, Clarendon
Press, 1997, 3; M. Heidenhain, European State Aid Law, Munich, Beck, \$1, no. 1). La CJUE parle également
d'une « interdiction » (par exemple, CJCE 10 déc. 1969, Commission c/ France, 6/69 et 11/69, Rec. I-00523 et
CJCE 22 mars 1977, Steinike \& Weinlig, 78/76, Rec. I-00595). D'autres auteurs, en revanche, soulignent la
différence entre l'interdiction et l'incompatibilité d'une aide d'Etat (par exemple, M. Waelbroeck \& A. Frignani,
Concurrence. Commentaire J. Mégret, Bruxelles, Editions de l'Université de Bruxelles, 1997, 334- 335, no 317
et L. Dubouis \& C. Blumann, Droit matériel de l'Union européenne, Issy-les-Moulineaux, LGDJ, 2015, 686) et
soutiennent donc que l'article 107, paragraphe 1, TFUE diffère d'une interdiction. ${ }^{134}$ A. Maitrot de la Motte, La concurrence fiscale dans la jurisprudence de la Cour de justice, in E. Carpano, M. Chastagnaret et E. Mazuyer (éd.), La concurrence réglementaire, sociale et fiscale dans l'Union européenne, Bruxelles, Larcier, 2016, 295-296 (voir aussi A. Maitrot de la Motte, Droit fiscal de l'Union européenne, Bruxelles, Bruylant, 2016, 52) ; I. Papadamaki, op. cit., 44 (en référence à M. Waelbroeck, Direct Taxation and EU law : integration or disintegration, EC Tax Review 2004, 2 et D. Berlin, Droit fiscal communautaire, Paris, PUF, 1988, 52); O. Peiffert, op. cit., par exemple 282, no. 502 et P.J. Wattel, O. Marres \& H. Vermeulen, op. cit., 45 et 46.

${ }^{135}$ En effet, si le non-exercice des compétences fiscales relevait du droit des aides d'Etat, les Etats membres seraient donc tenus d'exercer leurs pouvoirs fiscaux pour ne pas enfreindre le droit des aides d'Etat et seraient donc soumis à une obligation positive.

${ }^{136}$ A.C. Dos Santos, op. cit., 403.

${ }^{137}$ Communication de la Commission relative à la notion d' « aide d'État» visée à l'article 107, paragraphe 1, du traité sur le fonctionnement de l'Union européenne, JOUE 2016 C 262, p. 1, note 101.

${ }^{138}$ Cette position est prima facie soutenue par une analyse de fond de l'article 107, paragraphe 1 , du TFEU : après tout, cette disposition inclut les mesures « sous quelque forme que ce soit » et ne distingue pas selon les formes juridiques des interventions étatiques, mais « définit celles-ci en fonction de leurs effets » (par exemple, CJCE 2 juil. 1974, Italie c/ Commission, 173/73, Rec. 00709, point 33).

${ }_{139}$ Voir, par exemple, les affaires des fraises espagnoles (CJCE 9 déc. 1997, Commission c/ France, C-265/95, Rec. I-06959) et Schmidberger (CJCE 12 juin 2003, Schmidberger, C-112/00, Rec. I-05659), dans lesquelles la CJUE a effectivement jugé qu'une omission de la part de l'État membre de réagir à la conduite d'une entreprise privée entravant la libre circulation, peut constituer une violation de l'article 34 TFUE juncto article 4, paragraphe 3, du TUE.

${ }^{140}$ On pourrait se demander si le « détour » via l'article 4, paragraphe 3, du TUE est vraiment nécessaire pour créer des obligations positives pour les Etats membres en matière du droit des aides d'Etat (J. Baquero Cruz \& F. 
La planification fiscale agressive de la part des contribuables conduit à une réduction artificielle des charges (fiscales) normalement supportées par elles (voyez supra), ce qui est très proche à la définition ancienne d'une aide d'Etat (i.e. des mesures qui "sous des formes diverses, allègent les charges qui normalement grèvent le budget d'une entreprise ${ }^{141}{ }^{14}$ ), nous nous demandons donc si les Etats membres doivent prendre des mesures pour assurer que, dans leur systèmes fiscaux nationaux, ces avantages (par hypothèse, anormaux) ne soient pas obtenus par les contribuables concernés. Cependant, deux commentaires doivent être faits.

- Premièrement, l'obligation positive éventuelle à respecter est - selon la jurisprudence exposée ci-dessus - une obligation de résultat ${ }^{142}$, c.-à-d. les États membres restent libres de déterminer les moyens par lesquels cette obligation doit être respectée et «jouissent d'une marge d'appréciation pour déterminer quelles sont [...] les mesures les plus aptes $[\ldots] \gg{ }^{143}$. Appliqué à la question du traitement de la PFA, le droit des aides d'Etat pourrait donc seulement contraindre les Etats membres à adopter «des mesures nécessaires et appropriées $\rangle^{144}$, mais n'est pas capable de permettre à la Commission « de se substituer aux États membres pour leur prescrire les mesures qu'ils doivent adopter et appliquer ${ }^{145}$ contre la PFA ${ }^{146}$.

- Deuxièmement, puisque les destinataires (Normadressaten) du droit des aides d'Etat sont les États membres individuels, le comportement (action ou omission) d'un seul Etat membre doit être suffisant pour produire «l'illégalité » (en ce cas, l'octroi d'une aide d'Etat ; a contrario, «l'illégalité » ne peut résulter des comportements conjoints, c.-àd. de l'action ou omission d'un Etat membre en interaction avec celui d'un autre Etat

Castillo De La Torre, A note on PreussenElektra, EL Rev. 2001, 489 et suiv. ; contra, par exemple, P. Oliver, Some Further Reflections on the Scope of Articles 28-30 (ex 30-36) EC, Common Market Law Review 1999, 791 et J. Stuyck, Libre circulation et concurrence: les deux pilliers du marché commun, in M. Dony (dir.), M. Dony \& A. De Walsche, Mélanges en hommage à Michel Waelbroeck, Bruxelles, Bruylant, 1999, 1491). Bien qu'un tel «détour » est nécessaire pour créer des obligations pour les Etats membres dans le contexte des articles 101 et 102 , du TFUE (en sens que les Etats membres sont obligés de « ne pas prendre ou maintenir en vigueur des mesures [...] susceptibles d'éliminer l' effet utile des règles de concurrence applicables aux entreprises », voir par exemple, CJCE 21 sept. 1988, Van Eycke, 267/86, Rec. I-04769, point 16 ; CJCE 5 déc. 2006, Cipolla, C-94/04 et C-202/04, Rec. I-11421, point 46 et CJUE 23 nov. 2017, CHEZ Elektro Bulgaria, C-427/16 et C-428/16, publié(e) au Recueil numérique (Recueil général), point 41), c'est parce que ces dispositions s'adressent aux acteurs privés (et non aux Etats membres). Les dispositions relatives aux aides d'État, en revanche, sont destinées aux Etats membres. Par conséquent, l'article 107, du TFUE suffit à lui seul pour créer des obligations positives pour les Etats membres (J. Baquero Cruz \& F. Castillo De La Torre, op. cit., 489 et suiv. et CJCE 13 mars 2001, PreussenElektra, C-379/98, Rec. I-02099, points 64 et 65). Selon certains auteurs, un raisonnement similaire aurait dû s'appliquer dans les affaires les affaires des fraises espagnoles (CJCE 9 déc. 1997, Commission c/ France, C-265/95, Rec. I-06959) et Schmidberger (CJCE 12 juin 2003, Schmidberger, C-112/00, Rec. I-05659), l'article 34, du TFUE étant également adressé aux États membres (J. Baquero Cruz \& F. Castillo De La Torre, op. cit., 489 et suiv.).

${ }^{141}$ CJCE 23 févr. 1961, De Gezamenlijke Steenkolenmijnen in Limburg, 30/59, Rec. I-00003, 39.

142 Par exemple, L. Dubouis, La "guerre de la fraise »: l'impuissance publique sous la toise du droit communautaire. A propos de l'arrêt de la Cour de justice des Communautés européennes du 9 décembre 1997, Commission c/ République française, C-266/95, RFDA 1998, 120 et suiv. et K.J.M. Mortelmans, « Artikel 5 EG juncto artikel $30 \mathrm{EG}$ : een nieuwe route voor het vrije verkeer van goederen », AA 1998, no. 3, 207.

${ }^{143}$ CJCE 9 déc. 1997, Commission c/ France, C-265/95, Rec. I-06959, points 32-33.

144 Ibid.

145 Ibid.

146 Cette conclusion est proche de l'approche communautaire adoptée pour les infractions du droit de l'UE commises par des acteurs privés. Dans ces situations, la Cour a jugé que (i) les États membres sont tenus - sur la base du principe de coopération loyale (article 4, para. 3 TUE) - d'imposer des «sanctions effectives, proportionnées et dissuasives » pour assurer l'effectivité du droit de l'UE, sous réserve que (ii) les Etats membres est laissé le choix des mesures appropriées (par exemple, CJCE 21 sept. 1989, Commission c/ Grèce, 68/88, Rec. I-02965, point 24 ; CJCE 27 févr. 1997, Ebony Maritime, C-177/95, Rec. 1-1111, point 35 et CJUE 2 mai 2018, Scialdone, C-574/15, publié(e) au Recueil numérique (Recueil général), point 46). 
(membre $)^{147 / 148}$. Appliqué à la question de la PFA, cela veut dire que le droit des aides d'Etat ne couvre pas les avantages sélectifs fiscaux découlant des disparités ou des inadéquations entre les législations fiscales ${ }^{149}$; une position qui semble être acceptée dans l'affaire McDonald's (voyez supra).

La conclusion (prudente) selon laquelle le système de contrôle des aides d'État peut créer une obligation positive pour les États membres est nouvelle et quelque peu en contradiction avec l'opinion majoritaire dans la doctrine selon laquelle le système de contrôle des aides d'État est une forme d'intégration négative, ne donnant lieu qu'à des obligations négatives. D'une certaine manière, il semble donc que le système de contrôle des aides d'État pourrait générer des effets qui pourraient, contrairement à cette opinion majoritaire, ressembler à une forme d'intégration positive (c-à-d. le rapprochement des législations). Néanmoins, selon nous, les effets d'une telle obligation positive ne sont pas entièrement comparables à ceux du rapprochement des législations, les États membres n'étant pas tenus d'adopter des normes spécifiques et communes et, dans le même esprit, l'application du droit des aides d'État ne pouvant aboutir à la suppression des disparités entre les législations des États membres ${ }^{150}$.

\section{Chapitre 2. Structure horizontale}

Les procédures ouvertes par la Commission soulèvent également des questions liées à la répartition des compétences entre les institutions de l'UE. En effet, dans le cadre des recours en annulation concernant les récentes enquêtes de la Commission en matière de décisions fiscales, certains Etats membres ont fait valoir que de telles enquêtes constituent «une harmonisation fiscale déguisée $»$ par la Commission ${ }^{151}$. De même, divers auteurs ont estimé que les questions sous-jacentes aux enquêtes actuelles devraient être résolues par des modifications de la politique fiscale européenne qui doivent être introduites sous la forme de droit dérivé, excluant ainsi la possibilité d'un contrôle des aides d'Etat ${ }^{152}$.

\footnotetext{
${ }^{147}$ La question de savoir si une disposition du traité comporte des obligations pour des États membres individuels ou pour des États membres conjointement est une question de fond, qui dépend d'une interprétation de l'article 107, paragraphe 1, du TFUE. Bien qu'une telle analyse sort du champ de cette contribution, il convient de noter que cette position semble être soutenue par de nombreux auteurs, qui soutiennent que le droit des aides d'Etat (tout comme les dispositions relatives à la libre circulation) peut seulement supprimer des «manquements » des Etats membres au droit des aides d'Etat « lorsqu'elles sont causées par le comportement unilatéral d'une juridiction », c.-à-d. « pour lequel une (seule) juridiction peut être tenue pour responsable »; en d'autres termes, des « problèmes de deux juridictions » (par exemple, des disparités) ne peuvent pas être résolus par le droit des aides d'État (P.J. Wattel, O. Marres \& H. Vermeulen (dir.), o.c., 47-48; voir aussi, R. Szudoczky, The sources of EU law and their relationships: Lessons for the field of taxation, Université d'Amsterdam, Thèse, 2013, 526).

${ }^{148}$ Le fait que le recours en constatation du manquement (voir l'article 258, du TFUE) ne permette pas à la Commission de poursuivre plusieurs États membres à la fois (voir K. Lenaerts, I. Maselis \& K. Gutmann, EU Procedural Law, Oxford, Oxford University Press, 2015, no. 5.36) ne semble pas être lié à cette analyse. En plus, il semble que l'article 108 (qui est une lex specialis de l'article 258) du TFUE autorise la mise en œuvre de plusieurs États membres dans la même procédure (voir Décision 2010/606/UE de la Commission du 26 février 2010 concernant l'aide d'État C 9/2009 (ex NN 45/08, NN 49/08 et NN 50/08) mise à exécution par le Royaume de Belgique, la République française et le Grand-Duché de Luxembourg en faveur de Dexia SA, JOUE 2010 L 274, p. 54).

${ }^{149}$ Voyez en ce sens, par exemple, R. Luja, State Aid Benchmarking and Tax Rulings: Can We Keep It Simple?, in W. Schön \& E. Traversa, State Aid Law and Business Taxation, Berlin, Springer, 2016, 125.

${ }^{150}$ Voy en ce sens, O. Peiffert, op. cit., 284 et seq., no. 505 et seq.

151 Voy., par exemple, le troisième moyen invoqué par le Luxembourg dans sa demande en annulation de la décision Amazon (T-816/17) (« la Commission procèderait à une harmonisation fiscale déguisée en imposant sa propre interprétation du « bon » prix de transfert », nous soulignons).

${ }^{152}$ P. Lowe, op. cit., 913: « il est évident que les changements de politique fiscale à l'échelle de l'UE doivent être approuvés par des processus démocratiques normaux » (traduction libre). Voyez aussi T. Jaeger, Tax Concessions for Multinationals: In or Out of the Reach of State Aid Law?, Journal of European Competition Law \& Practice
} 
Cet argument peut cependant être compris de différentes manières. Premièrement, cela pourrait signifier que les dispositions relatives à l'harmonisation et aux aides d'Etat ont une portée mutuellement exclusive, ce qui signifie que si un domaine peut être ou a été harmonisé, il ne peut entrer dans le champ d'application du droit des aides d'Etat (cf. Section 1). Deuxièmement, cela pourrait impliquer que, même si les questions sous-jacentes pouvaient être traitées à la fois par les dispositions relatives à l'harmonisation et par celles relatives aux aides d'Etat, les premières devraient prévaloir (cf. Section 2$)^{153}$.

\section{Section 1. Champ d'application mutuellement exclusif ?}

Si le champ d'application des dispositions relatives aux aides d'Etat et celui des dispositions relatives à l'harmonisation s'excluent mutuellement, le contrôle des aides d'Etat ne devrait pas s'appliquer à des questions harmonisées ou harmonisables. En effet, si l'on acceptait cet argument sur la base d'un champ d'application mutuellement exclusif, le constat selon lequel l'harmonisation peut résoudre ou a résolu les problèmes en question impliquerait nécessairement que le droit des aides d'Etat ne le puisse pas. En d'autres termes, l'application du droit des aides d'Etat aux questions liées aux mesures fiscales directes des Etats membres serait exclue pour les mesures entrant dans le champ d'application des articles 115 à $117 \mathrm{du}$ TFUE. Cela signifierait que, comme certains aspects des compétences des Etats membres visà-vis de PFA ont été harmonisés par la directive sur la lutte contre l'évasion fiscale (ATAD ou « Anti-Tax Avoidance Directive » en langue anglaise) ${ }^{154}$, l'application du droit des aides d'Etat à ces questions serait discutable ${ }^{155}$.

L'argument selon lequel les deux ensembles de dispositions ont un champ d'application mutuellement exclusif bénéficie du soutien de certains auteurs, pour lesquels (i) la portée du contrôle des aides s'étend aux mesures nationales sélectives, tandis que (ii) l'harmonisation s'applique aux mesures générales (c'est-à-dire des mesures non sélectives) qui affectent le marché intérieur ou faussent la concurrence ${ }^{156}$. En d'autres termes, ces auteurs utilisent la notion de sélectivité pour distinguer le champ d'application du droit des aides d'Etat de celui des dispositions d'harmonisation ${ }^{157}$. Cette position se retrouve également dans la jurisprudence de la CJUE selon laquelle « le traité FUE instaure une délimitation précise entre, d'une part, les règles concernant les aides d'État $[. .$.$] et, d'autre part, les règles concernant les distorsions$ résultant de disparités entre les dispositions législatives, réglementaires ou administratives des

2017, 2: « il y a une ligne fine entre le caractère essentiellement répressif du contrôle des aides d'État [...] et une politique fiscale proactive pour le marché intérieur, réservée au législateur » (traduction libre).

${ }^{153}$ Enfin, cela pourrait également signifier que les dispositions relatives aux aides d'État ne peuvent pas produire d'effets s'apparentant à une harmonisation. Ce raisonnement a déjà été examiné supra.

${ }^{154}$ Directive (UE) 2016/1164 du Conseil du 12 juillet 2016 établissant des règles pour lutter contre les pratiques d'évasion fiscale qui ont une incidence directe sur le fonctionnement du marché intérieur, JOUE 2016 L 193, p. 114 et Directive (UE) 2017/952 du Conseil du 29 mai 2017 modifiant la directive (UE) 2016/1164 en ce qui concerne les dispositifs hybrides faisant intervenir des pays tiers, JOUE 2017 L 144, p. 1-11.

${ }^{155}$ Voir pour cet argument, si nous comprenons l'auteur correctement, D.S. Smit, Tax planning and State aid, in P. Pistone \& D. Weber (dir.), The Implementation of Anti-BEPS Rules in the EU: A Comprehensive Study, Amsterdam, IBFD, 2018, sec. 7.4.

${ }^{156}$ Par exemple, R. Szudoczky, The sources of EU law and their relationships: Lessons for the field of taxation, Université d'Amsterdam, Thèse, 2013, 360-366 (bien qu'elle reconnaisse que des chevauchements puissent exister, voir page 366); G.M. Roberti, op. cit., 404 et D. Waelbroeck, La compatibilité de systèmes fiscaux généraux avec les règles en matière d'aides d'État dans le traité CE, in X., Mélanges John Kirkpatrick, Bruxelles, Bruylant, 2004, 1025.

${ }^{157}$ O. Peiffert, op. cit., 294-295, nos. 527-530. 
États membres, et notamment leurs dispositions fiscales $[\ldots] »^{158}$. Sur cette base, on pourrait soutenir que l'adoption d'ATAD montre que le droit des aides d'Etat est inapplicable à certains questions de PFA.

Toutefois, comme l'a fait observer Peiffert, l'utilisation de la condition de sélectivité pour délimiter le champ d'application de la législation relative aux aides d'Etat et l'harmonisation semble discutable, car « aucune disposition du traité ne prévoit que la compétence du législateur doive être définie en fonction du droit des aides d'Etat. Les champs respectifs de ces deux pouvoirs sont construits de façon autonome ${ }^{159}$. À cet égard, il semble que le champ d'harmonisation ne soit pas limité aux mesures non sélectives ${ }^{160}$. En effet, même des mesures sélectives peuvent être traitées par l'harmonisation (et ce fut le cas dans les domaine nonfiscaux $\left.^{161}\right)^{162}$.

Par conséquent, le fait que certains aspects des compétences des Etats membres à l'égard de PFA ont été harmonisés par l'ATAD ne peut aboutir à la conclusion (a contrario) que de telles mesures sont des mesures générales et tombent donc hors du champ d'application du système de contrôle des aides d'Etat ${ }^{163}$.

\section{Section 2. Donner priorité à l'harmonisation à l'égard des aides d'Etat ?}

En acceptant la position selon laquelle la concurrence fiscale passive par les Etats membres peut être analysée à la fois dans le système de contrôle des aides d'Etat et par l'intégration positive grâce à l'harmonisation, l'argument selon lequel les problèmes sous-jacents en jeu devraient être résolus par voie d'harmonisation plutôt que par le droit des aides d'Etat pourrait être fondé sur la position selon laquelle le premier devrait avoir préséance. La justification d'un tel argument pourrait être que (i) les dispositions relatives à l'harmonisation constituent une lex specialis vis-à-vis des dispositions sur les aides d'Etat ou, à défaut, (ii) que l'harmonisation constitue un moyen moins intrusif d'atteindre le même résultat ou, finalement (iii) que les dispositions sur l'harmonisation constituent une base juridique plus appropriée.

Le premier argument, à savoir que les dispositions relatives à l'harmonisation constituent une lex specialis vis-à-vis du système de contrôle des aides d'Etat, ne peut, à notre avis, étayer cette affirmation. En effet, il semblerait qu'il n'y ait pas de relation hiérarchique entre les dispositions relatives aux aides d'Etat d'une part et les dispositions relatives au rapprochement des législations d'autre part. En outre, même si certains auteurs reconnaissent qu'une telle relation pourrait exister, ces auteurs soutiennent que c'est le système de contrôle des aides d'Etat qui constitue une lex specialis et « devrait avoir préséance sur les autres dispositions des

\footnotetext{
158 Trib. UE 11 juil. 2014, DTS Distribuidora de Televisión Digital c/ Commission, T-533/10, publié(e) au Recueil numérique (Recueil général), point 48 en référence à CJCE 13 janv. 2005, Streekgewest, C-174/02, Rec. I-00085, point 24.

${ }^{159}$ O. Peiffert, op. cit., 295, no. 530 et, de même, J. Bourgeois, Aides d'Etat, State aids, taxation measures and specificity. Some thoughts, in M. Dony \& A. De Walsche, Mélanges en hommage à Michel Waelbroeck, Bruxelles, Bruylant, 1999, 768.

${ }^{160}$ O. Peiffert, op. cit, 295 , no. 529.

${ }^{161}$ Voir dans le domaine du droit de l'environnement, ibid, avec des références.

${ }^{162}$ Certains auteurs ont fait valoir que, dans le scénario hypothétique où le TFUE n'aurait pas inclus de dispositions sur les aides d'État, les problèmes pourraient encore être résolus par l'harmonisation (J.-Y. Chérot, Les aides d'État dans les communautés européennes, Paris, Economica, 1998, 5-6, no. 11 et O. Peiffert, op. cit., 293 , no. 526).

${ }^{163}$ Cette question institutionnelle est différente de la question de fond de savoir si, lorsqu'un domaine juridique a été harmonisé, un Etat membre agissant dans ce domaine harmonisé peut être considéré comme octroyant une aide (par exemple lorsqu'il exécute simplement une obligation inscrite dans le droit dérivé).
} 
traités [UE] traitant des mesures générales émanant des Etats membres ${ }^{164}$, telles que les dispositions d'harmonisation.

Le deuxième argument, à savoir que l'harmonisation constitue un moyen moins intrusif d'atteindre le même résultat, semble également récolter peu de chance de succès. Il est vrai que le principe de proportionnalité guide les institutions de l'UE dans le choix des moyens permettant d'atteindre un certain résultat ${ }^{165}$. En effet, lorsqu'un résultat peut être obtenu par plusieurs moyens alternatifs, il faut choisir celui qui laisse le plus de liberté aux États membres ${ }^{166}$. En particulier, on pourrait soutenir que le principe de proportionnalité a été violé si une procédure en matière d'aide d'Etat ouverte par la Commission n'est pas nécessaire pour éviter une distorsion de concurrence sur le marché intérieur, mais vise principalement la réalisation d'un autre objectif ${ }^{167}$. Néanmoins, comme chaque action de la Commission dans le domaine des aides d'État contribue dans une certaine mesure à la promotion d'une concurrence non faussée sur le marché intérieur, le principe de proportionnalité ne pourrait que prévenir de véritables détournements de pouvoir ${ }^{168}$. En tant que tel, ce principe semble également avoir un impact limité à cet égard.

Le troisième argument, à savoir que les dispositions relatives à l'harmonisation constituent une base juridique plus appropriée, ne récolterait pas plus de succès. Cela est lié au principe de l'équilibre institutionnel ${ }^{169}$, qui est inclus dans l'article 13, paragraphe 2, du TUE TU $^{170}$ selon lequel «[c]haque institution agit dans les limites des attributions qui lui sont conférées dans les traités, conformément aux procédures, conditions et fins prévues par ceux-ci. Les institutions pratiquent entre elles une coopération loyale $»$. Le principe a également été décrit comme signifiant qu'une institution de l'UE ne peut pas empiéter sur les pouvoirs attribués dans le traité à une autre institution de $1^{\prime} U E^{171}$ et, dans ce sens, pourrait être à la base d'une affirmation selon laquelle la Commission perturberait cet équilibre institutionnel en entreprenant « une harmonisation fiscale déguisée » et de facto usurperait les compétences du Conseil (et du Parlement) dans l'adoption de mesures d'harmonisation des questions fiscales directes (articles 115-117 TFUE) ${ }^{172}$.

\footnotetext{
${ }^{164}$ J. Bourgeois, op. cit., $768-769$ (traduction libre).

165 Voir note 119.

${ }^{166}$ M. Peeters, op. cit., 167, no. 327 qui fait référence à G. Lienbacher, “Artikel 5 EGV” in J. Schwarze (dir.), $E U$ Kommentar, Baden-Baden, Nomos, 2009, 294, no. 44 et C. Calliess, "Art. 5 EGV” in C. Calliess \& M. Ruffert (dir.), EUV/EGV, München, Beck, 2007, 384, no. 57-58.

${ }^{167}$ M. Peeters, op. cit., 168, no. 328.

168 Ibid.

${ }^{169}$ Par exemple, M. Blanquet, op. cit., 83, no. 109: « la base juridique va permettre de déterminer l'équilibre des pouvoirs » et T. Ronse, op. cit., 269, no. 330. Pour une analyse récente du principe de l'équilibre institutionnel, voy. B. Bertrand, Le principe de l'équilibre institutionnel : la double inconstance, Europe 2016, n 6 , étude 5.

${ }^{170} \mathrm{La}$ CJUE a accepté que cette dernière disposition « traduit » le principe de l'équilibre institutionnel, voir CJUE 25 oct. 2017, Commission c/ Conseil, C-687/15, publié(e) au Recueil numérique (Recueil général), point 40 et CJUE, 28 juil. 2016, Conseil c/ Commission, C-660/13, publié(e) au Recueil numérique (Recueil général), point 32.

171 J.P. Jacqué, The Principle of Institutional Balance, CML Rev. 2004, 384 et K. Lenaerts \& A. Verhoeven, Institutional balance as a guarantee for democracy in EU governance, in C. Joerges \& R. Dehousse (dir.), Good Governance in Europe's Integrated Market, Oxford, OUP, 2002, 44-45.

${ }^{172}$ Comparez avec la conclusion de l'av. gén. Kokott dans C-66/14, Finanzamt Linz, point 113, dans lequel elle fait valoir, en substance, qu'une interprétation et application trop larges de l'article 107 et 108 du TFUE « risque de porter atteinte à $[\ldots]$ la répartition de la compétence dans l'Union entre le Parlement européen et le Conseil de l'Union européenne, conformément à l'article 14 TFUE, d'une part, et la Commission, conformément à l'article 17 TFUE, d'autre part ».
} 
Cependant, en plus d'être « un principe fragile avec un contenu incertain » ${ }^{173}$, «l'équilibre [institutionnel] auquel se réfère la Cour est donc celui établi par le traité ${ }^{174}$. En effet, « [a]insi conçu, on voit mal ce qui distingue le principe d'équilibre institutionnel d'un principe de légalité en vertu duquel les institutions doivent respecter les règles de compétences et de procédures du traité $»{ }^{175}$ Appliqué au cas présent, il semble que le principe de l'équilibre institutionnel ne peut pas servir de limite aux enquêtes récentes sur les aides d'Etat de la Commission. Comme l'a justement résumé Ibañez Colomo, « la lettre des dispositions - telle qu'interprétée par la Cour - est la seule limite significative à l'exécution par la Commission. Tant que l'application est basée sur une bonne compréhension des limites matérielles de ces dispositions, l'intervention est (légalement parlant) sans problème ${ }^{176}$. Par conséquent, « les arguments selon lesquels l'article 107, paragraphe 1, du TFUE est invoqué pour induire l'harmonisation de l'impôt sur les sociétés au sein de l'UE [...] ne seront pas couronnés de succès $»^{177}$.

\section{Conclusion}

Dans la première partie de cette contribution, les auteurs ont d'abord analysé comment, du point de vue institutionnel, la concurrence entre les États membres dans le domaine de la fiscalité directe a pu prospérer dans l'ordre juridique de l'UE. Par la suite, les auteurs ont établi que cette concurrence a dans une certaine mesure évolué d'une forme active de concurrence fiscale (c-àd. l'offre par un Etat membre d'un traitement fiscal avantageux au contribuable, à laquelle les contribuables réagissent en planifiant leurs activités en conséquence) vers une forme plus passive de concurrence fiscale (c-à-d. le manque de réaction ou la réaction inadéquate d'un Etat membre à des pratiques de planification fiscale agressive de la part des contribuables, dans le sens où les Etats membres ne neutralisent pas les avantages résultant d'une telle conduite par l'application d'une règle défavorable au contribuable).

Dans la deuxième partie de cette contribution, les auteurs ont premièrement analysé comment la Commission, utilisant ses prérogatives dans le cadre du système de contrôle des aides d'État, a agi contre les formes de concurrence fiscale passive des États membres. Par la suite, nous avons analysé si de telles actions de la Commission étaient fondées du point de vue du droit institutionnel de l'UE. À cet égard, nous avons adopté à la fois une perspective verticale (analysant la relation constitutionnelle entre les Etats membres et l'UE et en particulier les effets du droit des aides d'Etat sur la compétence des Etats membres en matière de la fiscalité directe) et une perspective horizontale (analysant les relations entre les institutions de l'ordre juridique de l'UE et en particulier la relation constitutionnelle entre les pouvoirs de l'UE en vertu du droit des aides d'Etat et le rapprochement des législations).

L'analyse des actions de la Commission d'un point de vue institutionnel présente un intérêt certain, de nombreux auteurs et parties demanderesse devant la CJUE ont soulevé de tels arguments. Cependant, sur la base de notre analyse, il apparaît qu'un certain nombre de ces

\footnotetext{
${ }^{173}$ S. Prechal, Institutional balance. A fragile principle with uncertain contents, in T. Heukels, N. Blokker \& M. Brus (dir.), The European Union after Amsterdam: a legal analysis, La Haye, Kluwer, 1998, 273 (traduction libre). 174 J.P. Jacqué, op. cit., 384 (traduction libre).

175 B. Bertrand, op. cit., no. 22

${ }^{176}$ P. Ibañez Colomo, The EU's Exclusive Competence in Competition Law, in S. Garben \& I. Govaere (dir.), The Division of Competences between the EU and the Member States: Reflections on the Past, the Present and the Future, Oxford, Hart Publishing, 2017, 126 (traduction libre). En outre, il semble que si l'on ne considère pas les dispositions relatives aux aides d'État comme conférant une compétence (voir supra), la jurisprudence de la Cour en matière de contrôle du choix de la base juridique ne s'applique pas (voir, par exemple, R.H. van Ooijk, De keuze der rechtsgrondslag voor besluiten van de Europese Unie, Kluwer, Deventer, 1999, 27-33).

177 Ibid.
} 
arguments ne semblent pas capables de limiter l'action de la Commission aux réactions des États membres face à la planification fiscale agressive des contribuables. Néanmoins, les auteurs ont pu identifier certains principes institutionnels (principalement dans la structure verticale de l'ordre juridique de l'UE) qui pourraient, en fait, influencer la manière dont la Commission devrait procéder, c'est-à-dire fournir un point de référence normatif pour évaluer les actions actuelles et futures de la Commission dans ce domaine. 